\title{
A Catalog of the Most Optically Luminous Galaxies at $z<0.3$ : Super Spirals, Super Lenticulars, Super Post-mergers, and Giant Ellipticals
}

\author{
Patrick M. Ogle (iD), Lauranne Lanz ${ }^{2}$ (D), Philip N. Appleton ${ }^{3}$ (D), George Helou ${ }^{3}$, and Joseph Mazzarella ${ }^{3}$ \\ ${ }^{1}$ Space Telescope Science Institute, 3700 San Martin Drive, Baltimore, MD 21218, USA; pogle@ stsci.edu \\ ${ }^{2}$ Dartmouth College, $\mathrm{NH}$, USA \\ ${ }^{3}$ IPAC, California Institute of Technology, Mail Code 220-6, Pasadena, CA 91125, USA \\ Received 2018 June 28; revised 2019 April 4; accepted 2019 April 8; published 2019 July 15
}

\begin{abstract}
We present a catalog of the 1525 most optically luminous galaxies from the Sloan Digital Sky Survey with $r$-band luminosity $L_{r}>8 L^{*}$ and redshift $z<0.3$, including 84 super spirals, 15 super lenticulars, 14 super post-merger galaxies, and 1400 giant ellipticals. With mass in stars of $10^{11.3}-10^{12} M_{\odot}$, super spirals and lenticulars are the most massive disk galaxies currently known. The specific star formation rates of super spirals place them on or below the star-forming main sequence. They must have formed stars at a high rate throughout their history in order to grow their massive, gigantic stellar disks and maintain their blue $u-r$ integrated colors. Their disks are red on the inside and blue on the outside, consistent with inside-out growth. They tend to have small bulge-to-total $(B / T)$ $r$-band luminosity ratios, characteristic of disk building via minor mergers and cold accretion. A large percentage of super disk galaxies (41\%) have double nuclei, double disks, or other signatures of ongoing mergers. Most (72\%) are found in moderate- to low-density environments, while the rest are found at the outskirts of clusters. It is likely that super spirals survive in these environments because they continue to accrete cold gas and experience only minor mergers at late times, by virtue of their enormous masses and angular momenta. We suggest that super postmergers are the product of super spiral major mergers and may be the precursors of some giant elliptical galaxies found in low-density environments. We present two new gravitational lens candidates in an appendix.
\end{abstract}

Key words: galaxies: elliptical and lenticular, cD - galaxies: spiral

Supporting material: machine-readable tables

\section{Introduction}

We recently found that $\sim 6 \%$ of the most optically luminous galaxies at redshift $z<0.3$ are giant, high-surface-brightness spiral galaxies, with masses of $10^{11}-10^{12} M_{\odot}$ and isophotal diameters of 55-140 kpc (Ogle et al. 2016). These super spiral galaxies are actively forming stars and appear to be vastly scaled-up versions of normal spiral galaxies. The extreme sizes, masses, and luminosities of super spirals extend the parameter space over which galaxy scaling laws may be studied, providing a new arena to test theories of massive galaxy formation and evolution.

In addition to giving new insights into galaxy formation and growth, super spirals can help discriminate among proposed mechanisms for quenching star formation in massive galaxies. The red optical colors of massive spiral galaxies may indicate that they are starved of gas and dying (Bamford et al. 2009; Masters et al. 2010; Schawinski et al. 2014). However, it is important to determine for any individual galaxy whether its red colors indicate low specific star formation rate (SSFR) or dust extinction via mid-infrared (MIR) photometry (Fraser-McKelvie et al. 2016). Star formation is thought to be quenched in most galaxies above a mass in stars of $\sim 10^{11} M_{\odot}$, by collisions, active galactic nucleus (AGN) activity, accretion shocks, or ram pressure stripping, which turn them into red and dead elliptical or lenticular galaxies (Dekel \& Birnboim 2006; Hopkins et al. 2006; Elbaz et al. 2007; Martig et al. 2009; Schawinski et al. 2014; Chang et al. 2015). Though they are rare, the existence of super spirals demonstrates that the limit to spiral galaxy size and mass is much higher than previously thought, and that high mass in stars cannot be the primary cause of star formation quenching. In fact, spiral galaxies with mass in stars $\sim 10^{11} M_{\odot}$ may be the most efficient at converting gas into stars, with mass fractions in stars approaching the cosmological baryon fraction (Posti et al. 2019). Super spirals and giant ellipticals may represent distinct evolutionary pathways for the most massive galaxies, depending on dark halo mass and angular momentum. Super spirals may remain unquenched because they reside in less massive dark halos than giant ellipticals of similar mass in stars.

Hydrodynamical simulations that include increasingly realistic star formation and AGN feedback prescriptions (Springel \& Hernquist 2005; Governato et al. 2007; Hopkins et al. 2009) show that galaxy mergers play a key role in determining the configuration, dynamics, star formation histories, and ultimate fates of galaxies. Simulations also reveal that typically half of the mass in stars is acquired via mergers, while the rest is formed in situ (Rodriguez-Gomez et al. 2016). A relatively large fraction of super spirals have two bulges surrounded by a common spiral disk or tidal features (Ogle et al. 2016), demonstrating the importance of mergers for these most massive disk galaxies. However, super spirals may survive most mergers because of their extremely large masses and sizes. That is, for a super spiral, even a merger with a typical $L^{*}$ galaxy is a high-mass-ratio, minor merger that will not destroy its massive disk.

This work extends the search for the most massive, optically luminous galaxies, relaxing the restriction that they be detected in the near-ultraviolet (NUV) band by the Galaxy Evolution Explorer (GALEX), with the goal of understanding how super spirals relate to other types of massive galaxies. We present a new class of super lenticular galaxies that may be quenched super spirals and a new class of super post-merger galaxies that may be the product of super spiral major mergers. 

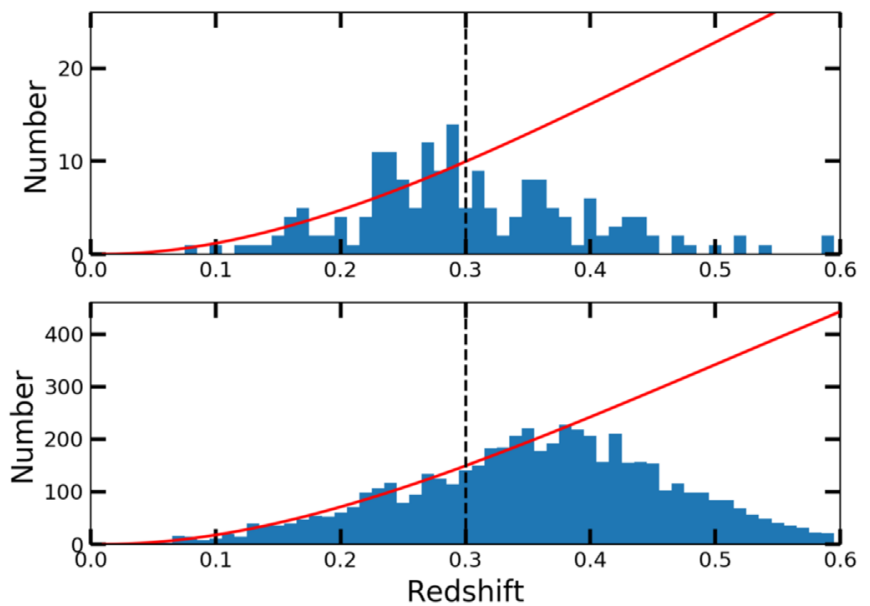

Figure 1. Redshift distributions for galaxies with $L_{r}>8 L^{*}$. Top: super disk (spiral and lenticular) redshift histogram compared to a constant comoving density curve. Bottom: giant elliptical redshift histogram. The Ogle et al. Galaxy Catalog is limited to $z<0.3$ (dashed line). The distribution for ellipticals extends to higher redshift because they are augmented by the SDSS luminous red galaxy sample.

\section{Sample and Photometry}

\subsection{Sample Selection}

Our galaxy sample is primarily selected for high Sloan Digital Sky Survey (SDSS) $r$-band luminosity $\left(L_{r}>8 L^{*}\right)$, which is an excellent tracer of mass in stars for unobscured galaxies. Unlike Ogle et al. (2016), here we do not impose requirements of GALEX NUV-band detection or spiral morphology, resulting in a sample that also includes lenticular, elliptical, and peculiar galaxies, and which is less biased toward high SFRs. We selected all galaxies from the NASA/IPAC Extragalactic Database (NED) with an existing spectroscopic redshift $z<0.3$ and $r$-band photometry from SDSS I or II (York et al. 2000; Strauss et al. 2002). These galaxies were then ranked by their $r$-band monochromatic luminosities $\left(L_{r}\right)$, after correcting for Galactic extinction and applying a $K$-correction. We report $L_{r}$ relative to the characteristic luminosity at the knee of the galaxy luminosity function of $L^{*}=$ $5.41 \times 10^{43} \mathrm{erg} \mathrm{s}^{-1}$ at $6200 \AA$ (Blanton et al. 2003).

The redshift distribution for spiral galaxies with $L_{r}>8 L^{*}$ cuts off at $z=0.30 \pm 0.02$ (Figure 1), corresponding to the SDSS I/II spectroscopic selection limit of $r=17.77$ (Strauss et al. 2002). The distribution of elliptical galaxies cuts off at a higher redshift of $z=0.38 \pm 0.01$, corresponding to the effective redshift cutoff of the SDSS luminous red galaxy (LRG) sample (Eisenstein et al. 2001). Therefore, to mitigate against incompleteness and selection effects at greater redshifts, we restrict the present study to SDSS galaxies with $L_{r}>8 L^{*}$ at $z<0.3$.

We found 1616 candidate galaxies at $z<0.3$ with $L_{r}>8 L^{*}$, presented as the Ogle et al. Galaxy Catalog (OGC: Tables 2-5). We visually inspected the SDSS three-color images to determine their morphologies and checked their redshifts against their SDSS spectra. Galaxies were classified as spiral, lenticular, elliptical, or peculiar, based on visual appearance. In particular, relative prominence of bulge and disk components and presence of spiral arms were the key discriminants. We give a breakdown of OGC galaxy types in Table 1. A total of 1525 galaxies are legitimate high-luminosity galaxies. The remaining 91 galaxies that have inaccurate photometry (51 in
Table 1

OGC Galaxy Type Percentages

\begin{tabular}{lccc}
\hline \hline Type & Subtype & Number & Percentage \\
\hline Super disk & & 99 & 6.5 \\
\hline & Spiral & 84 & 5.5 \\
& Lenticular (S0/Sa) & 15 & 1.0 \\
\hline Giant elliptical & 1400 & 91.8 \\
Post-merger & 14 & 0.9 \\
Bright AGN & 12 & 0.8 \\
\hline Luminous galaxies & 1525 & 100.0 \\
Rejects & 91 & \\
\hline All & 1616 & \\
\hline
\end{tabular}

Table 3), or incorrect redshifts (24 in Table 4), or that overlap with foreground objects (16 in Table 5) are excluded from further analysis.

Apart from constructing a manageable sample of the most optically luminous galaxies, there is no particular physical significance to our $8 L^{*}$ cutoff. As we shall demonstrate, super spirals are dramatically scaled-up versions of much more abundant $L^{*}$ spirals, albeit with some significant structural, photometric, and other differences. Since our $8 L^{*}$ limit is somewhat arbitrary, the search for superluminous, giant spiral galaxies could be extended down to lower luminosities. For example, there are 16,301 SDSS galaxies with $L_{r}>5 L^{*}$ at $z<0.25$. Using this $5 L^{*}$ cutoff would yield galaxies in the top $2 \%$ of the $r$-band luminosity distribution, compared to the top $0.2 \%$ for our adopted $8 L^{*}$ cutoff.

\subsection{Super Spirals}

We find 84 super spiral galaxies with $L_{r}>8 L^{*}$ at $z<0.3$ (Table 6; Figures 11 and 12). This includes 32 new super spirals, augmenting our original sample of 53 (Ogle et al. 2016). Inspection of an archival Hubble Space Telescope (HST) image (see Appendix A.1) led us to remove OGC 0302, reducing the sample size from 85 to 84 .

In order to compare morphologies (Figure 2), we crossmatched the OGC with the Galaxy Zoo DR1 catalog (Lintott et al. 2011). We find that only $22 / 84$ super spirals $(26 \%)$ are classified as spirals with $P_{\text {spiral }}>0.8$ by Galaxy Zoo, 10 are classified as ellipticals (12\%), 50 are classified as uncertain (60\%), and two (OGC 0574 and 586) are not classified (2\%). Super spirals with uncertain Galaxy Zoo classification include galaxies that appear to have normal spiral morphologies, such as OGC 0065 and $0713\left(P_{\text {spiral }}=0.57\right.$ and 0.60$)$, and spirals that are disturbed by mergers, such as OGC 0789 and 1304 $\left(P_{\text {spiral }}=0.61\right.$ and 0.29). It appears that the Galaxy Zoo classifications of many super spirals are rendered uncertain by their relatively high redshifts (Bamford et al. 2009) and high merger fraction.

The mean number density of super spirals in our sample is $58 \mathrm{Gpc}^{-3}$ within a comoving volume of $7.14 \mathrm{Gpc}^{3}$, corrected for the $20.3 \%$ sky coverage of SDSS II. This is $5.5 \%$ of the total number density of high-luminosity galaxies in our sample $\left(1050 \mathrm{Gpc}^{-3}\right)$. Correcting for an inclination incompleteness of $40 \%$ (Section 5), the number density of super spirals increases to $97 \mathrm{Gpc}^{-3}, 9.2 \%$ of the population of galaxies with $L_{r}>8 L^{*}$. 


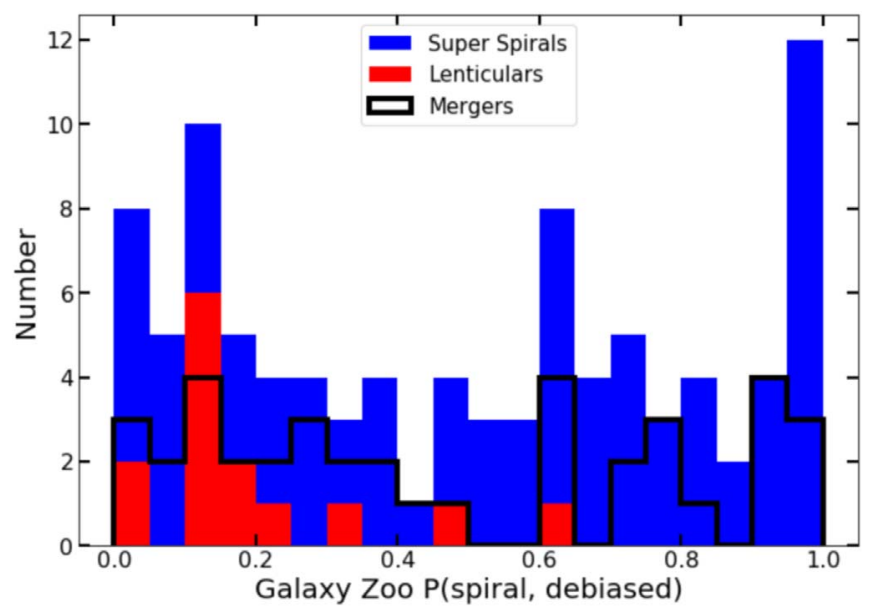

Figure 2. Galaxy Zoo 1 debiased spiral probability (Bamford et al. 2009), corrected for redshift bias. Because the redshift bias corrections are large for galaxies at $z>0.08$, and spiral galaxies at these redshifts may be misclassified as early type, Galaxy Zoo morphologies should be interpreted with caution. Total bar height indicates total spirals and lenticulars, while color represents the fraction of each type. The black histogram shows the number of mergers, regardless of morphological type.

We emphasize that super spiral galaxies are one of the rarest galaxy populations in the universe. Their comoving number density is a factor of $10^{3}-10^{4}$ lower than samples of massive galaxies constructed from smaller surveys at higher redshift (e.g., Davari et al. 2017; Faisst et al. 2017). Existing deep surveys are not large enough to yield significant numbers of super spirals. Deeper large surveys by Euclid, the Wide Field Infrared Survey Telescope, and the Large Synoptic Survey Telesope will be necessary to discover and characterize super spiral progenitors at $z>1$.

Extending our search for high-luminosity SDSS galaxies to $0.3<z<0.6$ yields 83 additional super spirals (included in Figure 1 but not tabulated). The redshift distribution of super spirals falls off at $z>0.3$, corresponding to the SDSS I/II magnitude limit for spectroscopy and our $L_{r}>8 L^{*}$ luminosity lower limit.

\subsection{Super Lenticulars and Giant Ellipticals}

Ogle et al. (2016) predicted a new class of super lenticular galaxies, but for the most part they were excluded from their sample due to an NUV-band selection criterion. Here we identify 15 super lenticular (S0/Sa) galaxies with $L_{r}>8 L^{*}$ at $z<0.3$ (Table 6; Figures 11 and 12). It is easiest to identify lenticulars at intermediate inclination (Section 5), where they are less likely to be confused with ellipticals or edge-on spirals. Based on the small number in our sample, the space density of super lenticulars is $>10 \mathrm{Gpc}^{-3}$. It is likely that we are missing $>60 \%$ of super lenticulars, at both low and high inclination, based on the distribution of observed inclinations (Section 5).

There are 1400 giant elliptical galaxies in the OGC, which constitute $91.8 \%$ of the sample. Their mean comoving number density is $970 \mathrm{Gpc}^{-3}$, corrected for SDSS II sky coverage. The most optically luminous giant elliptical galaxy is OGC 0021 (2MASX J12220526+4518109), with $L_{r}=19.8 L^{*}$. It resides at the center of a rich galaxy cluster, which is frequently (but not always) the case for the giant ellipticals in our sample.

\subsection{Super Post-mergers and Luminous AGN Hosts}

There are 14 peculiar galaxies $(0.9 \%$ of the sample) that have disturbed morphologies, indicative of recent mergers (Table 6 and Figure 13). We highlight one additional peculiar starburst galaxy (OGC 1662, $L_{r}=7.9 L^{*}$ ) which does not quite make our $8 L^{*}$ cutoff but has a very peculiar morphology and multi-hued appearance. We include all of these galaxies in our photometric analysis, but differentiate them from galaxies with more regular morphologies.

We exclude 12 non-spiral galaxies ( $0.8 \%$ of the sample) that are quasar hosts, blazar hosts, or contain bright stars seen in projection from further analysis because an AGN or foreground star is likely to contribute a significant fraction of the luminosity at wavelengths of interest (Table 7 and Figure 13).

\subsection{Photometry}

We use CModel catalog photometry from SDSS DR6 (York et al. 2000) and aperture photometry from the Two Micron All Sky Survey (2MASS; Skrutskie et al. 2006) and the Wide-field Infrared Survey Explorer (WISE; Wright et al. 2010) to estimate the total optical luminosities, mass in stars, and SFRs. The smallest 2MASS and WISE apertures that encompass the $D_{25}$ isophotal diameter at $r=25$ mag were selected. The SDSS CModel magnitudes are effectively aperture-matched and are therefore appropriate for measuring integrated galaxy color. All galaxies are detected in the $\operatorname{SDSS} u, g, r, i, z$ bands. A total of 90 super disk galaxies are detected in the 2MASS $K_{\mathrm{s}}$ band and 86 in the $W 3$ band. Of the 1400 giant ellipticals, 127 are undetected in the $K_{\mathrm{s}}$ band, and 128 are undetected in $W 3$.

We correct SDSS and 2MASS photometry for Galactic extinction using the NED extinction calculator, based on the Galactic extinction maps of Schlafly \& Finkbeiner (2011). We $K$-correct SDSS and 2MASS magnitudes to rest-frame values with a simple, custom procedure that performs log-linear interpolation of the observed spectral energy distribution (SED). We $K$-correct WISE magnitudes using two representative model SEDs, one for star-forming galaxies with $W 2-W 3>2$, and a second for quiescent galaxies with $W 2-W 3 \leqslant 2$, yielding $K$-corrections of $K(W 3)<0.33 \mathrm{mag}$ and $K(W 2-W 3)<0.21$ over the redshift range of our sample.

\section{Mass in Stars and SFR}

The SEDs of nearly all galaxies in our sample are dominated by stellar populations at NUV to NIR wavelengths, and warm dust emission at MIR wavelengths. Galaxies with SEDs dominated by AGNs (0.8\% of the sample) are excluded from this analysis. We estimate mass in stars (Figure 3) from $K$-corrected 2MASS $K_{\mathrm{s}}$ magnitude and $K$-corrected SDSS $u-r$ color, using the prescription of Bell et al. (2003), with a small correction $(+0.004$ dex $)$ to convert to a Chabrier (2003) initial stellar mass function. The mass estimates are relatively insensitive to both dust extinction and variations in mass-tolight ratio $M / L$ with mean stellar population age.

The super spiral masses are on average four times larger than those given by Ogle et al. (2016), primarily because of a systematic error they made converting from $K_{\mathrm{s}}$-band monochromatic luminosity to solar luminosity units. The $K_{\mathrm{s}}$ monochromatic luminosity was incorrectly divided by the solar bolometric luminosity, rather than the solar $K_{\mathrm{s}}$-band monochromatic luminosity, which is a factor of 5.5 smaller. This is partially offset by the new $K$-corrections to the $K_{\mathrm{s}}$ band magnitudes, 

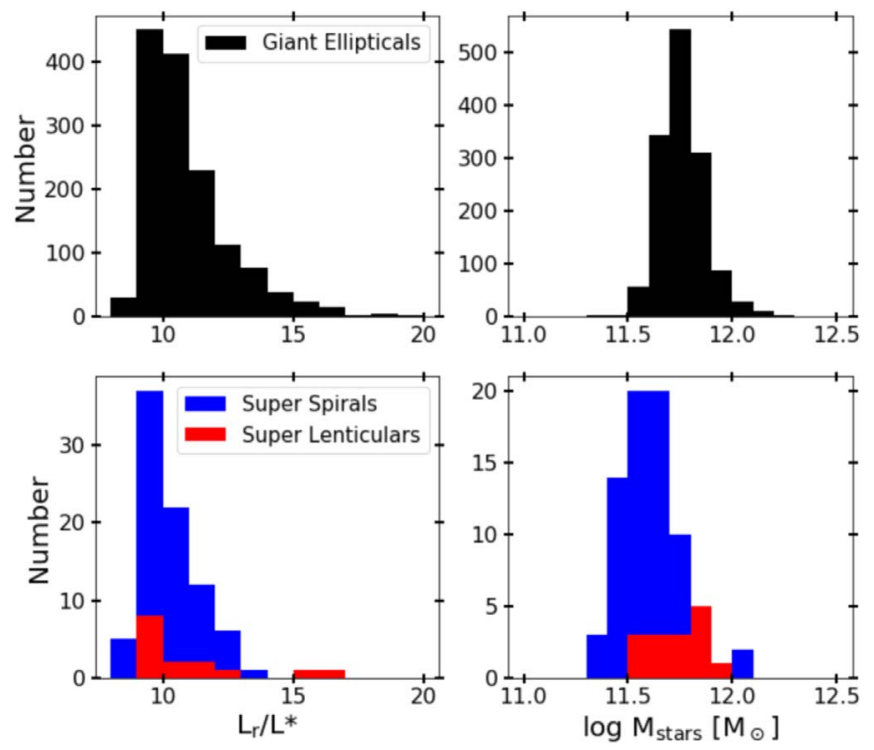

Figure 3. Luminosity and mass of giant ellipticals compared to super disks Left: $r$-band luminosity distributions. Right: distributions of mass in stars.

which also act to reduce the scatter in the distribution of masses in stars. These new, more accurate estimates lead to a better understanding of how truly massive super spirals are. They in turn affect the SSFRs and location of super spirals relative to the star-forming main sequence (SFMS; Figure 4), shifting them to the right by 0.6 dex and placing most of them on or below this relation.

We compare the masses in stars of super disk galaxies and giant ellipticals in Figure 3. The narrow range of high $r$-band luminosities in the OGC effectively selects for the most massive galaxies, with masses in stars of $10^{11.3}-10^{12.3} M_{\odot}$. The mean mass in stars is a factor of 1.4 (0.14 dex) smaller for super spirals than for super lenticulars and giant elliptical galaxies, because of their systematically lower stellar $M / L \mathrm{~s}$. A major merger between a super spiral and another massive galaxy could potentially create super lenticular and giant elliptical galaxies of greater mass. Super spiral major mergers may provide a channel to create giant elliptical galaxies outside of galaxy clusters, like the most massive isolated elliptical OGC 0078 (2MASX J02295551 +0104361 ), with $\log M_{\text {stars }}=11.9$. However, the dark halo masses of isolated giant ellipticals like this one are likely to be considerably smaller than the dark halo masses of giant ellipticals in clusters, even though they have comparable masses in stars. The very different evolutionary histories of ellipticals in the most massive halos versus those that form from super spiral mergers may potentially result in different fractional mass in stars, gas content, kinematics, and morphology.

We estimate SFRs from $K$-corrected WISE W3-band $(12 \mu \mathrm{m})$ fluxes, which trace warm dust heated by UV photons in starforming galaxies (Chang et al. 2015). ${ }^{4}$ We plot SFR against mass in stars for OGC galaxies in Figure 4. This method gives consistent SFR values for star-forming galaxies compared to full-SED fitting with MAGPHYS (da Cunha et al. 2008; Ogle et al. 2016) and is relatively insensitive to dust extinction,

\footnotetext{
4 Note that there is a known offset of -0.22 dex (Chang et al. 2015) between SFRs derived from WISE measurements compared to those derived from $\mathrm{H} \alpha$ fluxes (Brinchmann et al. 2004; Elbaz et al. 2007). We adopt the SFMS of Elbaz et al. (2007), shifted downward by -0.22 dex to match WISEestimated SFRs.
}

compared to SFRs estimated from UV or $\mathrm{H} \alpha$ emission. On the other hand, old stellar populations contribute most of the W3-band luminosity for quiescent elliptical galaxies with the lowest SSFRs (Bressan et al. 2006; Chang et al. 2015), so their SFRs should be considered to be upper limits. Between these two extremes, both old and young stellar populations will contribute to the W3 luminosity. Assuming a baseline WISE color of $W 2-W 3=0.32$ for quiescent ellipticals, the break-even point (equal W3 luminosity from star formation and old stars) occurs at $W 2-W 3 \simeq 0.32+2.5 \log 2=1.07$ (Figure 5). Only seven super spirals, three super lenticulars, and one super post-merger have $W 3-W 2$ colors bluer than this, such that their $W 3$ luminosity is dominated by old stellar populations. Dust heated by A-type stars in recently quenched galaxies may also lead to an overestimate of the SFR (Alatalo et al. 2014, 2017). This is not the case for most super disk galaxies in our sample, though it may be a concern for the peculiar, post-merger galaxies, depending on the fractions of UV photons from A stars and star-forming regions.

The super spirals and super lenticulars have SFRs ranging from 1 to $30 M_{\odot} \mathrm{yr}^{-1}$ (Figure 4). Their SSFRs span a broad range from $(0.02-1.5) \times 10^{-10} \mathrm{yr}^{-1}(1 / \mathrm{SSFR}=6-500 \mathrm{Gyr})$, with most falling on or below the SFMS, extrapolated from less-luminous SDSS spiral galaxies. There appears to be a continuum of massive, super disk galaxies, from active starforming super spirals to quiescent super lenticulars, similar to the well-established sequence for $L^{*}$ galaxies. There is no sharp dividing line between super spirals and super lenticulars in the $M_{\text {stars }}$-SFR plane. Because super spirals are so massive, not even those with the highest SFRs and IR luminosities are global starbursts. Based on SDSS spectra, we do find strong nuclear starbursts in five super spirals (OGC 0217, 0454, 1457, 1464, and 1520) and relatively strong $\mathrm{H} \alpha$ from nuclear star formation in two (OGC 1312, and 1512).

The giant ellipticals are for the most part quiescent, with $\mathrm{SSFR}<1.5 \times 10^{-11} \mathrm{yr}^{-1}$. While a small number (14) have greater SSFRs than this, they are not starbursts. Six of these (OGC 0034, 0087, 0123, 0792, 1412, and 1581) have high Balmer line equivalent widths and significant populations of young, blue stars in their SDSS spectra. The dust and UV emission from the vast majority of more quiescent giant ellipticals may indicate a low level of ongoing star formation accompanied by young stellar populations.

Most of the super post-mergers $(11 / 14)$ have SSFRs that formally put them on the SFMS. However, they have SDSS spectra with high $\mathrm{H} \delta$ equivalent widths characteristic of dominant A-star populations in post-starburst galaxies. The W3-band fluxes may therefore have a significant contribution from warm dust heated by UV emission from post-starburst stellar populations (Melnick \& De Propris 2013; Alatalo et al. 2017). NUV emission from A-F-type stars can linger for 1-3 Gyr following starburst activity, leading to significant MIR emission. The post-mergers display a range of $\mathrm{H} \alpha$ equivalent widths from star formation and AGN activity, indicating that they are still forming stars and feeding their black holes. Only one (OGC 0792) has an SDSS spectrum that indicates an ongoing burst of nuclear star formation. Five have clear AGN signatures in their SDSS spectra, including two (OGC 0247 and 1413) with high-luminosity AGNs that contribute significantly to their $W 3$-band luminosity and red $W 1-W 3$ colors. We may be viewing most of these peculiar galaxies during quenching episodes immediately following gas-rich 


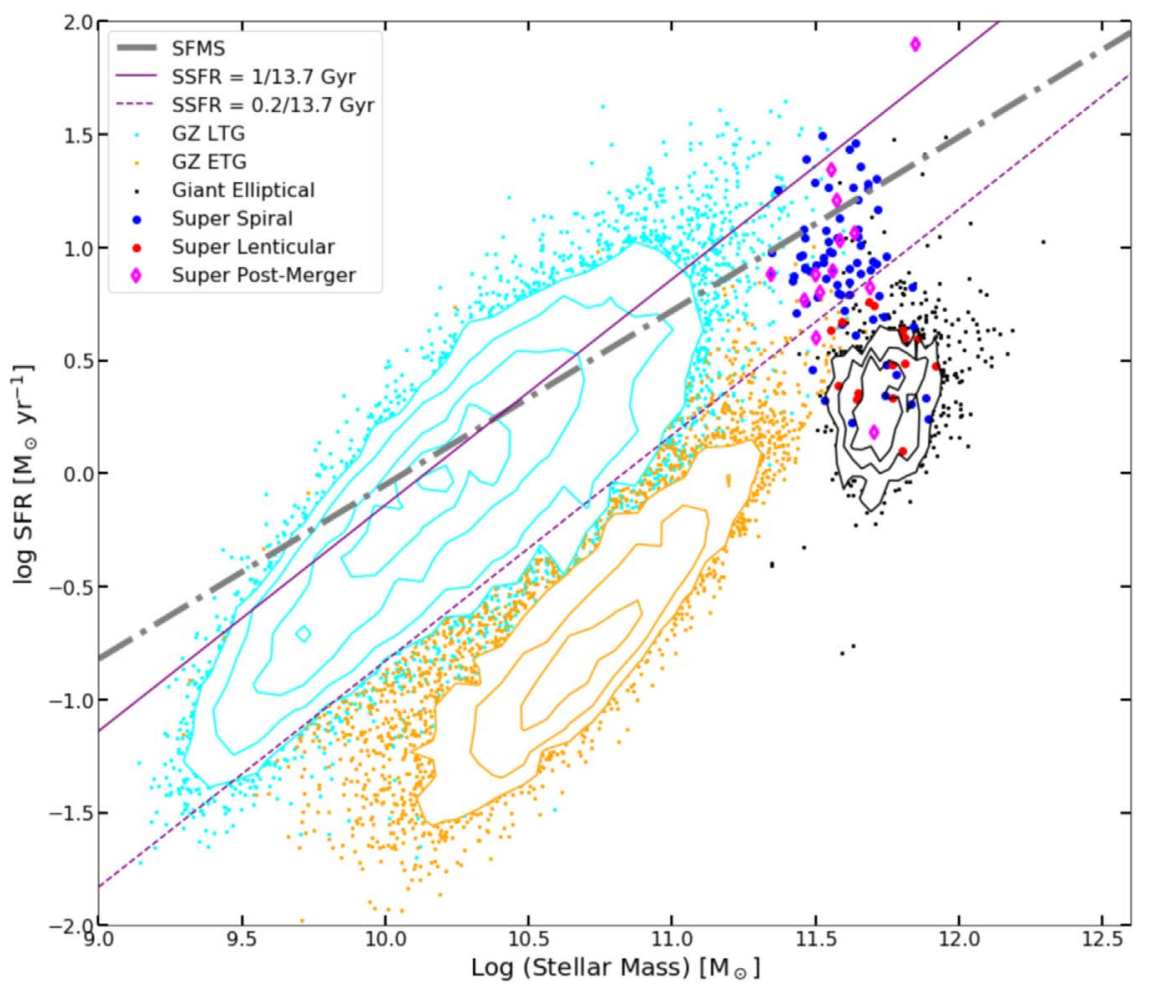

Figure 4. Star formation rates (SFRs) and masses in stars of super disks, post-mergers, and giant ellipticals compared to Galaxy Zoo late-type and early-type galaxies (GZ LTGs and ETGs: Alatalo et al. 2014; Schawinski et al. 2014; see the legend). The thick dotted-dashed line indicates the star-forming main sequence at $z \sim 0$ (Elbaz et al. 2007), which has been shifted downward by 0.22 dex to match the WISE-based SFR estimates of Chang et al. (2015). The dashed and solid purple lines indicate specific SFRs of $(0.2-1) /(13.7 \mathrm{Gyr})$, a range where active star-forming galaxies would double their masses in stars in one to five times the current age of the universe.

mergers, where the observed AGN activity may eventually clear out much of the remaining gas. If all of the star-forming super post-mergers in our sample are the result of super spiral major mergers, we estimate a per-galaxy super spiral destruction rate of $0.16 \mathrm{Gyr}^{-1}$, assuming a post-merger settling timescale of $1.0 \mathrm{Gyr}$ (Lotz et al. 2008). The two super postmergers (OGC 0743 and 1141) with SDSS spectra and SEDs characteristic of predominantly old stellar populations may be the product of dry mergers.

\section{Colors}

\subsection{Total System Colors}

For lower-mass galaxies with $M_{\text {stars }}=10^{9}-10^{11} M_{\odot}$, there is a well-established bimodal distribution in color between blue, star-forming spiral galaxies and red elliptical galaxies with low SFR (Schawinski et al. 2014). Galaxies that fall in the so-called green valley that separates the two populations are primarily disk galaxies (spirals and lenticulars) with relatively low specific SFRs, plus a small contingent of elliptical galaxies with modest SFRs (Figure 5). The origin of the bimodal color distribution has been attributed to various star formation quenching mechanisms, including galaxy mergers and quasar activity.

Most super spirals are blue, with rest frame $u-r<2.2 \mathrm{mag}$, similar to less-massive Galaxy Zoo late-type galaxies (Lintott et al. 2008). The WISE $W 2-W 3$ color tracks SSFR, to the extent that it correlates with $K_{\mathrm{s}}-W 3$, the basis of our SFR versus mass plot (Figure 4). Super spirals with red $W 2-W 3$ color have high SSFR, while lenticulars have relatively low SSFR and bluer $W 2-W 3$ color. We find a linear anti-correlation between $W 2-W 3$ and $u-r$ for our sample of massive galaxies (Figure 5(a)), reflecting an anti-correlation between SSFR and luminosity-weighted mean stellar population age. Galaxies with the highest SSFR have blue $u-r$ and red $W 2-W 3$, characteristic of luminous, young stellar populations, while galaxies with lower SSFR have redder $u-r$ and bluer $W 2-W 3$ color, from a mix of young and old stellar populations. This stands in contrast to lower-mass disk galaxies along the blue sequence, which follow a dog-leg trajectory in color space (Figure 5(a)). The $W 2-W 3$ color for these galaxies remains red even after they have quenched and moved into the green valley, perhaps because of dust heated by a post-starburst population of A stars (Alatalo et al. 2014, 2017). Super spirals do not appear to follow this trajectory in color space, indicating a different evolutionary history that is consistent with ongoing star formation and a mixture of old and young stellar populations. We can reproduce the observed range of SDSS and WISE colors by a linear mix of a young, star-forming stellar population $(u-r=1.4, W 2-$ $W 3=3.8)$ with an old, quiescent stellar population $(u-r=2.65$, $W 2-W 3=0.32$ ), where we vary the star-forming mass fraction $f_{\mathrm{sf}}$ (Figure 5(a)). This allows us to estimate the star-forming mass fraction for any given galaxy, and the fraction of light in each band that comes from the two stellar populations. This model does not apply to quenching galaxies, such as lower-mass lenticulars, which follow a different trajectory in this color space.

While super spirals have similar colors to less massive spirals, they stand dramatically apart in the color-mass plane (Figure 5(b)). Most super spirals have blue $u-r$ colors corresponding to high SSFRs, in spite of their enormous mass in stars. In order to better understand the stellar populations of 

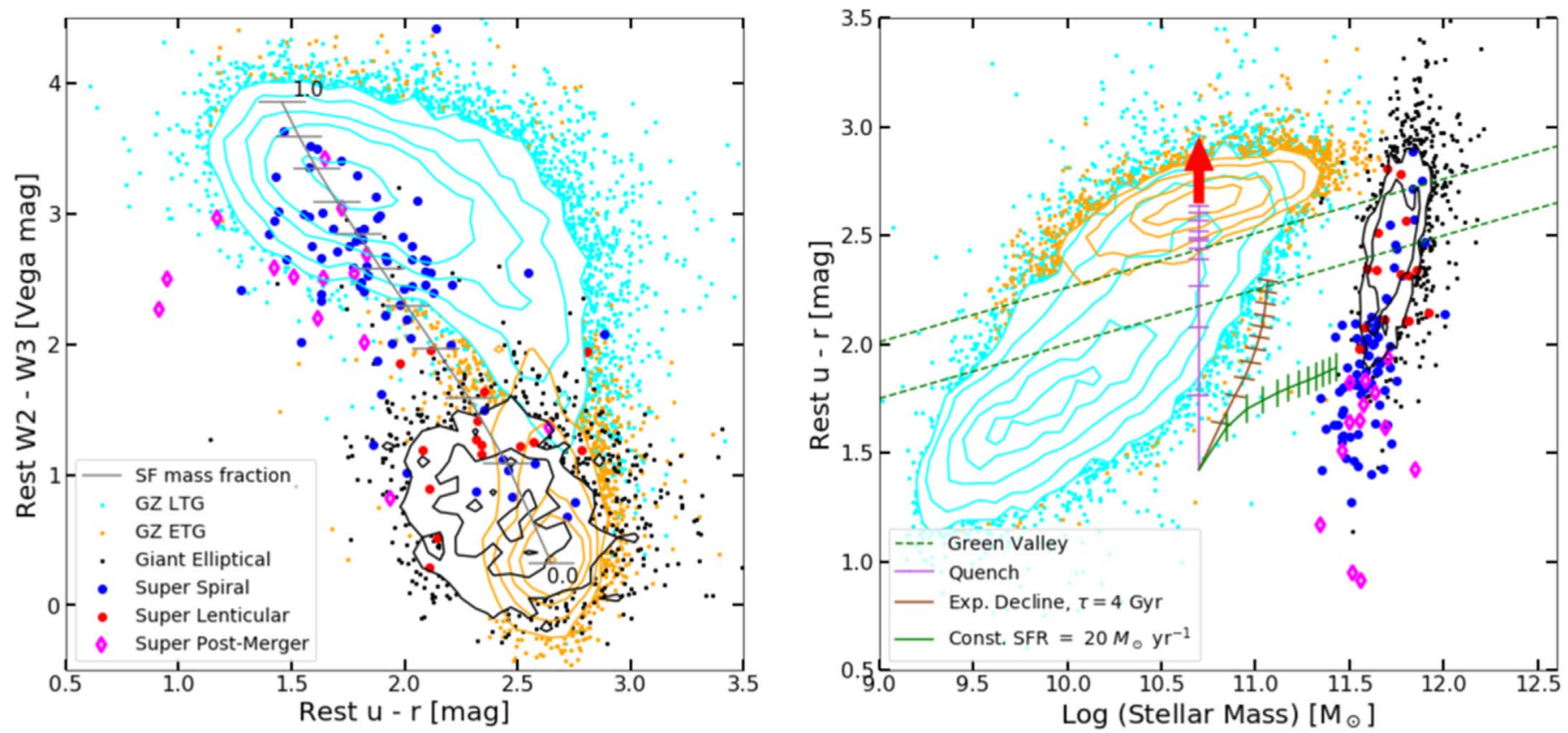

Figure 5. (a) SDSS and WISE colors of super spirals (dark blue), super lenticulars (red), super post-mergers (magenta diamonds), and giant ellipticals (black) compared to Galaxy Zoo (GZ) classified SDSS galaxies at $z=0.02-0.05$ (Lintott et al. 2008; Alatalo et al. 2014; Schawinski et al. 2014). Reddening by Galactic-type dust would increase $u-r$, with no effect on the WISE color. The gray track follows the linear mixing of a young, star-forming stellar population $(u-r=1.4$, $W 2-W 3=3.8)$ with an old, quiescent stellar population $(u-r=2.65, W 2-W 3=0.32)$. The star-forming fraction $f_{\text {sf }}$ by $W 2$ luminosity, which follows mass in stars, is indicated by the tick marks separated by 0.1 . The mix with $f_{\mathrm{sf}}=0.1$ has $W 2-W 3=1.09$ and equal contributions to $W 3$ luminosity from quiescent and starforming populations. (b) SDSS color-mass diagram, without any correction for internal extinction. The location of the green valley (green dashed lines), as determined for less massive galaxies by Schawinski et al. (2014), is shown for comparison. We over-plot evolutionary tracks based on Bruzual \& Charlot (2003) stellar population synthesis models, with a starting age of $0.5 \mathrm{Gyr}$ and markers spaced $1 \mathrm{Gyr}$ apart (tick marks at color ages of $1.5,2.5, \ldots 11.5 \mathrm{Gyr}$ ). All three galaxies start at $z=6$ with $\log M_{\text {stars }}=10.7$, solar metallicity $Z=Z_{\odot}$, and a stellar population age of $0.5 \mathrm{Gyr}$, which represents the newly formed bulge. In the quench model (lavender track), the galaxy quenches immediately. For the other two models, a disk subsequently forms with either constant SFR (green track) or exponentially declining SFR (brown track) with $e$-folding timescale of $\tau=4$ Gyr. Galaxy $u-r$ color gets redder with time even for the constant SFR model as old stellar populations accumulate. The endpoint mass, color, and bulge/disk mass ratio of the constant SFR model is similar to super spirals in our sample. The red arrow indicates the effect on $u-r$ of increasing the end-point metallicity by 0.2 dex to $1.6 Z_{\odot}$.

super spirals and lenticulars, we created synthetic galaxy colors by summing the stellar population synthesis (SPS) spectral models of Bruzual \& Charlot (2003). We assume solar metallicity $\left(Z=Z_{\odot}\right)$ for the massive galaxies in our sample. The influence of the mass-metallicity relation in star-forming galaxies (e.g., Tremonti et al. 2004) on $u-r$ is also examined by increasing the end-point metallicity by 0.2 dex to $1.6 Z_{\odot}$, the maximum value supported by the SPS models. For a constant or declining SFR, the $u-r$ color reddens monotonically with time, yielding a range of color that matches super spirals (Figure 5(b)). In particular, galaxies that form stars at a constant rate become steadily redder with time as old stars accumulate within their disks, reaching $u-r=1.8 \mathrm{mag}$ after $12 \mathrm{Gyr}$. Galaxies that are redder than this must have declining SFRs. Increasing the metallicity by 0.2 dex to $Z=1.6 Z_{\odot}$ reddens $u-r$ by $0.25 \mathrm{mag}$ in our SPS models.

Both super lenticulars and massive ellipticals are on average redder than super spirals, consistent with older stellar population ages. For a single stellar population created in a $\delta$-function burst, the $u-r$ color increases from $u-r=1.4$ mag at $t=0.5 \mathrm{Gyr}$ to $u-r=2.7 \mathrm{mag}$ at $t=11.5 \mathrm{Gyr}$ in our SPS quench model (Figure 5(b)). This type of evolution describes passive galaxies on the red sequence that formed and quenched not long after the big bang. We introduce the color age $t_{\text {color }}$, appropriate to such a single burst stellar population, in order to characterize the average-luminosity-weighted stellar population ages of galaxies. The giant ellipticals have a median $u-r$ color of $2.5 \mathrm{mag}$, indicating $t_{\text {color }} \sim 5.5 \mathrm{Gyr}$, compared to less-massive Galaxy Zoo early-type galaxies that have a median $u-r$ color of $2.7 \mathrm{mag}$ and $t_{\text {color }} \sim 11.5 \mathrm{Gyr}$. The bluer colors and younger color ages of the massive ellipticals in our sample may indicate that they are more susceptible to bouts of renewed star formation, perhaps as a result of more frequent mergers or cooling flows in dense environments (Egami et al. 2006; Burns et al. 2008).

\subsection{Color Gradient}

The disks of spiral galaxies typically display a negative color gradient, with bluer colors at larger radii, attributed to a combination of decreasing stellar population age and decreasing metallicity with radius (de Jong 1996; Bell \& de Jong 2000). While a gradient in dust extinction can also in principle cause a color gradient, this would require an unrealistically large optical depth and dust scale height (de Jong 1996).

We performed elliptical aperture photometry on the SDSS images of one of the brightest, nearby super spirals (OGC 0543) to characterize its radial color profile (Figure 6). The integrated, $K$-corrected color over the full galaxy is $u-r=$ $2.03 \mathrm{mag}$, typical for the massive star-forming galaxies in our sample. The $r$ - and $i$-band radial light profiles, which track mass in stars, are smoother and drop more quickly than the $u$-band profile, which tracks star formation. Oscillations in the $g$-band surface brightness correspond to spiral arms in the stellar disk. The $u-r$ color is bluer in the nucleus than in the 

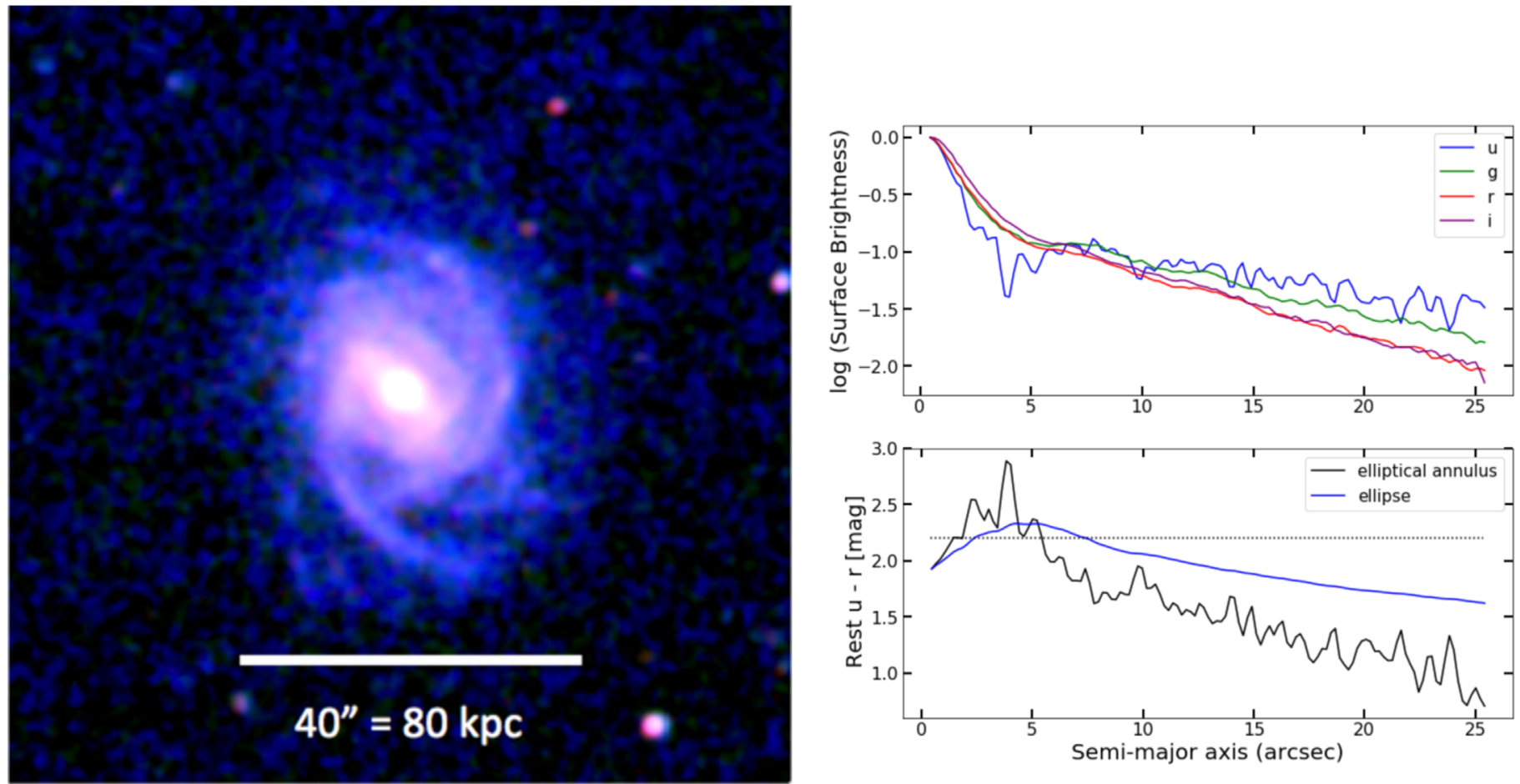

Figure 6. Image: super spiral OGC 0543 SDSS gri 3-color image. Top plot: surface brightness profile in SDSS bands, within elliptical annuli, normalized to unity at $r=0^{\prime \prime}$. Bottom plot: $K$-corrected $u-r$ color (solid black line) decreases with radius in the disk, indicating increasing specific SFR and decreasing color age or decreasing metallicity. The integrated $u-r$ color within an elliptical aperture (solid blue line) also becomes bluer as the aperture size is increased. The optical color separator for the massive blue- and red-sequence galaxies in our sample $(u-r=2.2 \mathrm{mag})$ is indicated by the dotted line.
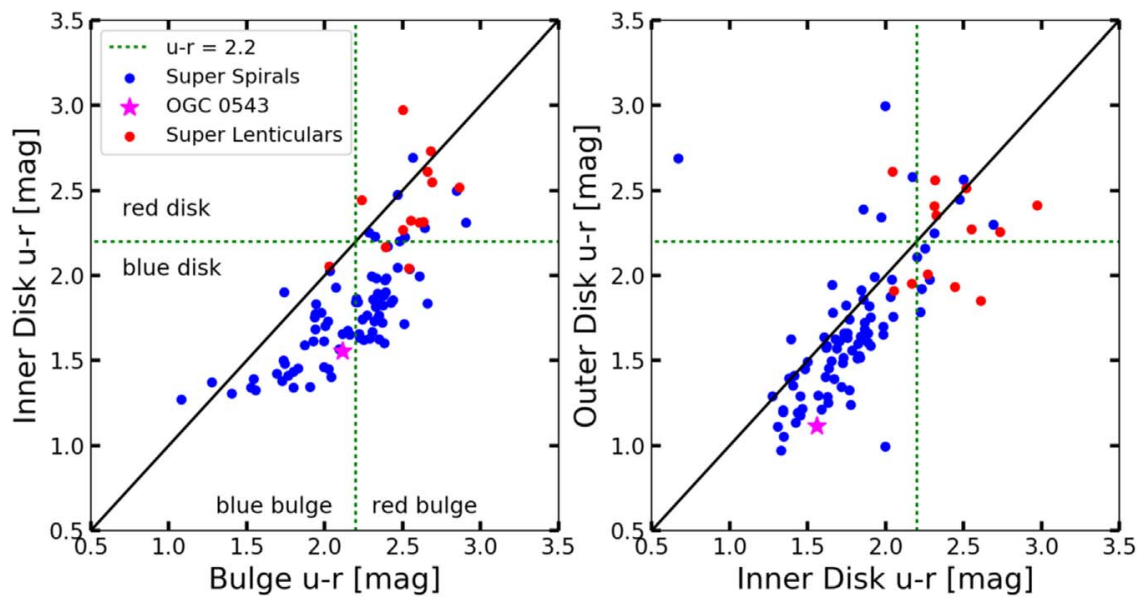

Figure 7. Radial $u-r$ color gradients for super spirals (blue points) and super lenticulars (red points). Left: the inner disks (middle one-third elliptical annulus) of super spirals and super lenticulars are systematically bluer than their bulges (inner one-third ellipse). Right: the outer disks (outer one-third annulus) of super spirals are systematically bluer than their inner disks (middle one-third elliptical annulus), indicating younger stellar populations. The diagonal (black line) delineates equal $u-r$ color. The horizontal and vertical dotted lines at $u-r=2.2 \mathrm{mag}$ separate red and blue stellar populations. The data point for our super spiral case study subject, OGC 0543 (Figure 6) is plotted as the star symbol.

inner disk because of AGN activity (OGC 0543 has a Seyfert 1 nucleus). Outside of the nucleus, the $u-r$ color gets progressively bluer with increasing radius in the disk, indicating increasing SSFR and decreasing color age with radius. The large range in $u-r$ color seen in the disk of OGC 0543 spans the full range of integrated $u-r$ color for spiral galaxies (Figure 5(b)). The $u$-band surface brightness profile indicates a current SFR that declines gradually (by only $0.5 \mathrm{dex})$ from $6^{\prime \prime}$ to $25^{\prime \prime}(12-60 \mathrm{kpc})$. The exponential $r$-band radial profile over the same interval indicates that a larger surface density of old stars has accumulated in the inner disk over cosmic time, relative to the outer disk. This is consistent with inside-out growth via gas accretion (possibly accompanied by some inward migration of old stars).

We measured the surface brightness and color profiles in elliptical annuli at fixed PA for all super spirals and super lenticulars in our sample, to see if they show similar color gradients to OGC 0543. We summarize our results by comparing the integrated $u-r$ color inside three annuli, with outer semimajor axes of $0.33,0.66$, and 1.0 times the isophotal radius at $r=25 \mathrm{mag}, R_{25}$ (Figure 7). We label these three regions bulge, inner disk, and outer disk, though it should be 

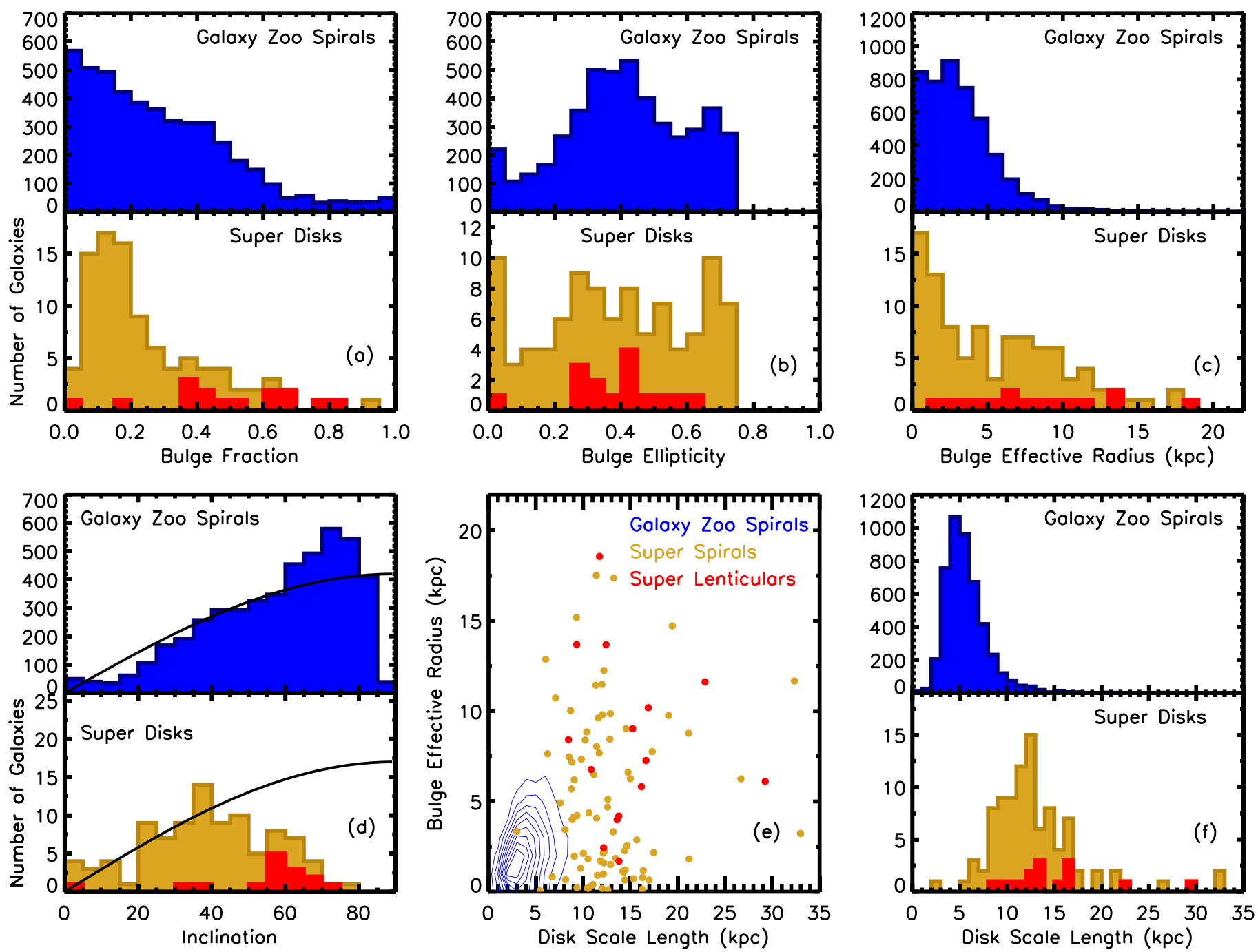

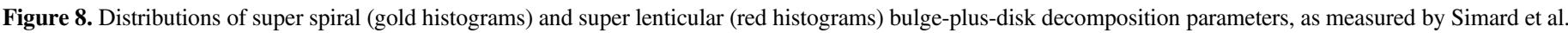

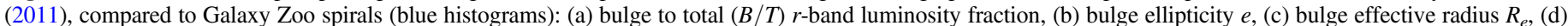

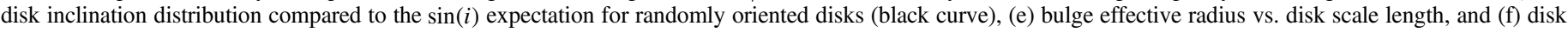
exponential scale length.

kept in mind that all three regions may contain flux contributions from the bulge, pseudo-disk, disk, or stellar bar.

As expected, the inner disks of super spirals are bluer than their bulges (Figure 7(left)). Super lenticulars have systematically redder bulges and disks than super spirals, consistent with older stellar populations and lower SSFR. We can divide super disk galaxies (spirals and lenticulars) into three categories, based on the $u-r$ colors of their inner disks and bulges, which can be tied to a range of different star formation histories. Forty-five percent have blue disks and blue bulges, $42 \%$ percent have blue disks and red bulges, and $13 \%$ percent have red disks and red bulges. The first category corresponds to galaxies with star-forming disks and bulges, the second to starforming disks and quiescent bulges, and the third to quiescent disks and bulges. The very bluest bulges (possibly pseudobulges) with $u-r<1.7$ mag have color ages of $<1.5 \mathrm{Gyr}$, indicating recent star formation. Red disks with $u-r>2.2$ mag and color ages $>3.5$ Gyr have quenched star formation, including both lenticulars and red spirals like those found in Galaxy Zoo (Bamford et al. 2009).
The outer disks of super spirals are on average 0.2 mag bluer in $u-r$ compared to their inner disks (Figure 7(right)). This is consistent with the color gradient found for OGC 0543 above, confirming a tendency for a younger stellar population age (or lower metallicity) in the outer disks of super spirals. Although still quite red, the inner disks of super lenticulars are systematically bluer than their bulges, consistent with younger color ages and later quenching times. Their outer disks show no systematic difference in $u-r$ color with respect to their inner disks.

\section{Bulge-Disk Decomposition}

We use GIM2D bulge--disk decompositions from Simard et al. (2011) to compare the quantitative morphologies and sizes of super spirals and super lenticulars to Galaxy Zoo spirals (Figure 8). In these decompositions, the bulge is fit by an $n=4$ Sérsic profile with ellipticity $e$ and effective radius $R_{e}$, while the disk is fit by an exponential profile with scale length $R_{d}$ and inclination $i$. We confirm the results of Ogle et al. (2016) with our larger sample. 
The mean bulge-to-total $r$-band luminosity ratio $(B / T)$ is smaller for super spirals than for Galaxy Zoo spirals (Figure 8(a)). The super spiral $B / T$ distribution peaks at $B / T=0.1-0.2$, while there is no clear peak in the $B / T$ distribution for Galaxy Zoo spirals. The $B / T$ distribution of Galaxy Zoo spirals has a tail with $B / T>0.7$, where the disk contributes a minor fraction of the $r$-band flux. Only one super spiral (OGC 1514) falls in this part of the distribution. Otherwise, our visual morphological identification of super spirals is consistent with $B / T$ values indicating a large disk component. All but two super lenticulars (OGC 0044 and 1386 ) have $B / T>0.35$, consistent with a major merger origin.

The distribution of bulge ellipticity (Figure 8(b)) is similar for super spirals and Galaxy Zoo spirals. Many super spirals $(20 \pm 5 \%)$ appear to have bars (Table 6). While the bar fraction is low compared to the total bar fraction of $65 \%$ for $L^{*}$ spiral galaxies at $z=0.14-0.47$ measured with the HST (Sheth et al. 2008 ), it is consistent with the strong bar fraction of $27 \%$ for the same set of galaxies (dropping to $20 \%$ for spirals with the smallest $B / T$ ). The lower spatial resolution of SDSS compared to HST may cause us to miss the weak bars in our sample. Higher spatial resolution imaging is needed to improve on our measurement of the bar fraction in super spirals. The mean bulge ellipticity for barred super spirals is $\langle e\rangle=0.50 \pm 0.08$, compared to $\langle e\rangle=0.38 \pm 0.02$, for non-barred super spirals. It is likely that the presence of a bar increases the fit bulge ellipticity in some cases.

Both bulges and disks are on average larger in super spirals and super lenticulars than in Galaxy Zoo spirals (Figures 8(c), (e), (f)). The disk scale lengths of super spirals extend to much larger values than those of Galaxy Zoo spirals. The distributions peak at $R_{d}=12.5 \mathrm{kpc}$ and $R_{d}=4.5 \mathrm{kpc}$, respectively. The distribution of bulge effective radius also extends to much larger values for super disks than for Galaxy Zoo spirals. However, a significant fraction of super spirals (36/99) have small, unresolved bulges with $R_{e}<2 \mathrm{kpc}$ in spite of their large disk scale lengths $(>5 \mathrm{kpc})$. Both super spirals and super lenticulars cover a large range in $R_{e} / R_{d}$, perhaps reflecting a range in merger histories and merger mass ratios.

Even though we dropped the NUV-band selection criterion of Ogle et al. (2016), we still find a large deficit of super spirals at inclinations $i>50^{\circ}$, compared to both Galaxy Zoo spirals and the expected distribution for randomly oriented disks (Figure 8(d)). Roughly $40 \%$ of super spirals must have dropped out of the parent sample because dust extinction in their highly inclined disks caused their $r$-band luminosities to fall below our selection threshold of $L_{r}>8 L^{*}$. An additional extinction of $\Delta r=0.6$ mag would suffice to move the brightest face-on super spiral (OGC 0065) below our luminosity selection threshold. The most luminous edge-on spiral galaxy in SDSS $\mathrm{I} / \mathrm{II}$ at $z<0.3$ is $2 \mathrm{MFGC} 12344(z=0.1407)$, with inclination $i=81^{\circ}$. Its dust lane crosses just above its nucleus, consistent with its high inclination (Figure 9). Its apparent luminosity $\left(L_{r}=7.9\right)$ is just below our sample selection threshold, and its $r$-band isophotal diameter $(120 \mathrm{kpc})$ rivals the largest super spiral in our sample (OGC 0139: $D=134 \mathrm{kpc}, L_{r}=13.4$ ). Selection by NIR luminosity may help to recover many more of these edge-on, dust-obscured super spirals. Super lenticulars are preferentially selected at intermediate inclinations of $50^{\circ}$ $75^{\circ}$, because face-on lenticulars are difficult to distinguish from ellipticals, and because edge-on lenticulars may be misclassified as spirals. We attribute the similar excess of

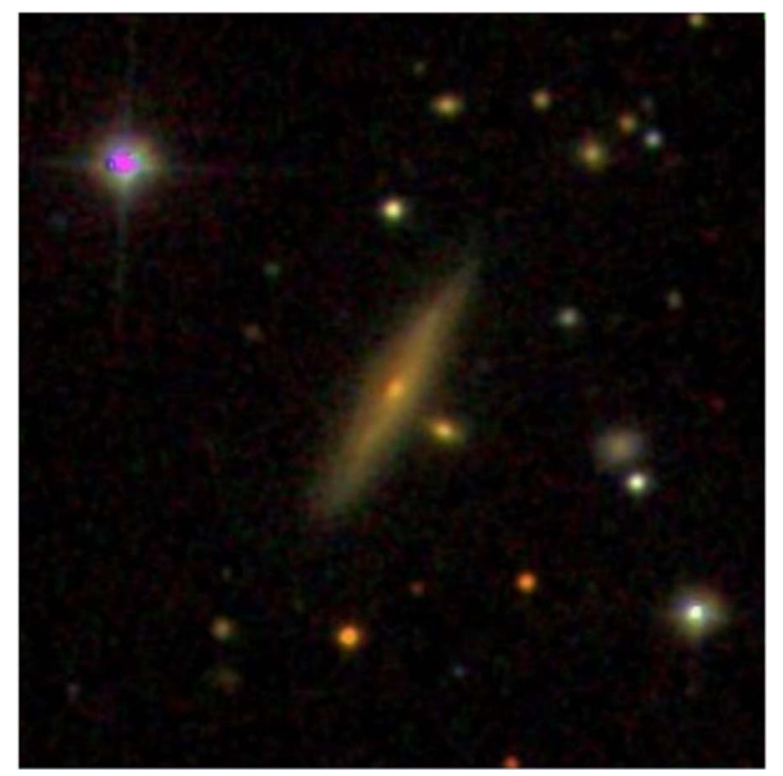

Figure 9. SDSS image of 2MFGC 12344, the most luminous nearly edge-on spiral galaxy in SDSS I/II. The field of view is $250 \mathrm{kpc}$ on a side.

Galaxy Zoo spirals at inclinations of $60^{\circ}-80^{\circ}$ to a population of lenticulars.

\section{Environment}

Most super spirals and super lenticulars are found in moderately dense environments (Figures 14 and 15), with an average of $7.8 \pm 0.3$ SDSS galaxies within a projected radius of $150 \mathrm{kpc}$. In comparison, OGC giant ellipticals have on average roughly twice as many galaxies within the same radius $(14.5 \pm 0.1)$. We find only eight super spirals in dense environments with 14 or more apparent companions within a projected radius of $150 \mathrm{kpc}$ : OGC 0299, 0586, 0799, 1023, 1304, 1329, 1457, and 1559. While most companions are more than 1 magnitude fainter than the super spiral, some are potentially massive enough to result in a major, disruptive merger (e.g., companions to OGC 0299, 0799, 1304, and 1457).

We searched for known galaxy clusters and groups within $2^{\prime}$ of each super spiral, using NED (Table 8 ). We find that $28 \%$ of super spirals and super lenticulars appear to be associated with clusters or groups of galaxies (Table 8). For these, we used the NED Environment Search tool to count the number of galaxies with separations and redshifts that put them within $1 \mathrm{Mpc}$ and $500 \mathrm{~km} \mathrm{~s}^{-1}$ (N1) or within $10 \mathrm{Mpc}$ and $5000 \mathrm{~km} \mathrm{~s}^{-1}$ (N10). While these numbers give a rough sense of cluster richness, they must be quite incomplete for galaxies at the highest redshifts. Most cluster or group members would not be luminous enough to make it into the SDSS spectroscopic sample. Indeed, it is seen that clusters associated with the lowest redshift super spirals have the most SDSS redshifts (e.g., 310 for OGC 1559 at $z=0.186$ ), while candidate clusters associated with super spirals at the highest redshifts have fewer SDSS redshifts (e.g., 23 for OGC 044 at $z=0.293$ ). Deeper redshift surveys are necessary to measure the richness and velocity dispersions of the highest-redshift candidate clusters.

For four super spirals in the richest clusters (OGC 0345, 0516,1268 , and 1304), we generated velocity plots with NED Environment Search (e.g., Figure 10). The locations of the super spirals within the velocity distributions confirm that they 

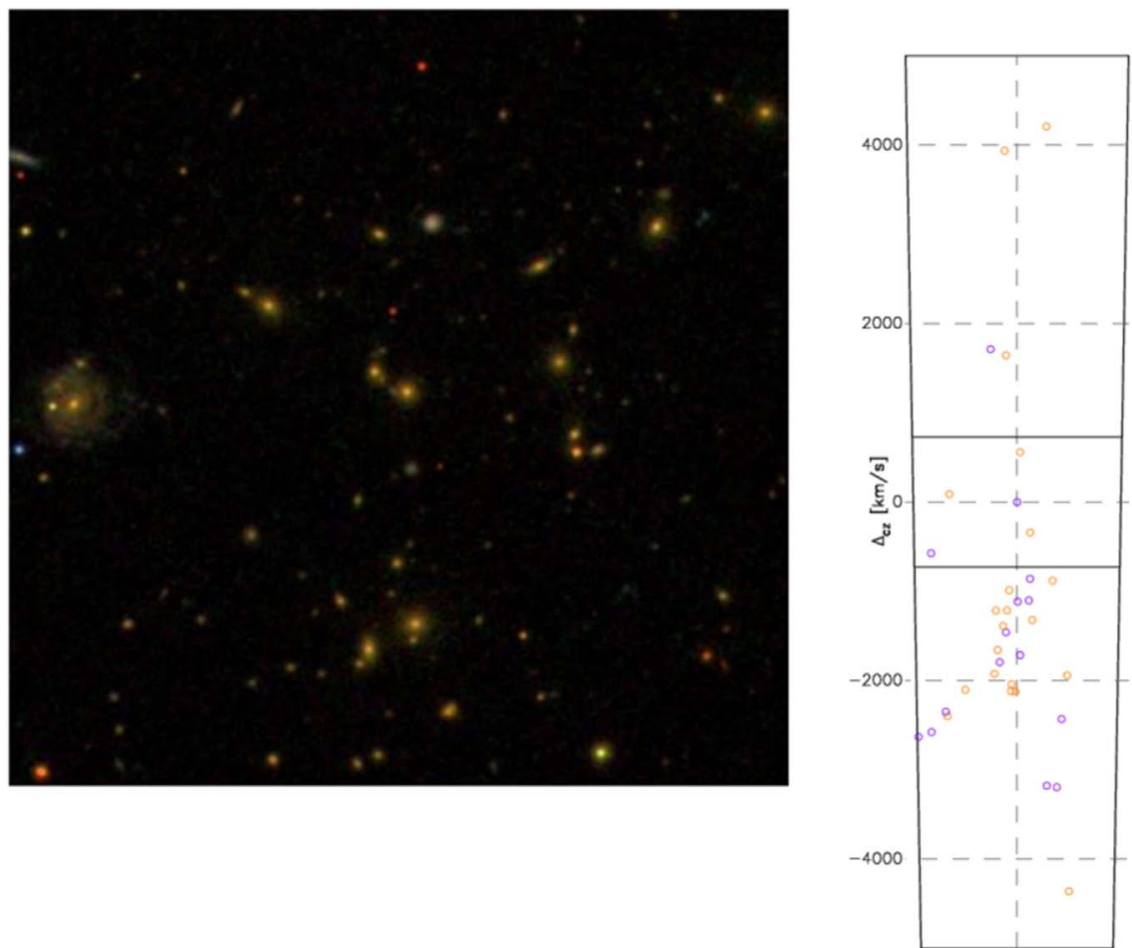

Figure 10. Left: super spiral OGC 0345 (at left edge) in galaxy cluster WHL J092608.1+240524. The SDSS image is $200^{\prime \prime}=716 \mathrm{kpc}$ on a side. Right: positionvelocity diagram from NED Environment Search, $10 \mathrm{Mpc}$ wide and centered on OGC 0345 . Orange and purple correspond to galaxies above and below the plane of OGC 0345, respectively.

are either cluster or supercluster members. The large velocity dispersions of these clusters (full range of $\pm 3000 \mathrm{~km} \mathrm{~s}^{-1}$ ) indicate that they are very massive. However, no super spirals reside at cluster centers, and their velocities relative to the mean cluster redshift are large $\left(\sim 2000 \mathrm{~km} \mathrm{~s}^{-1}\right)$. This indicates that these super spirals did not form at the cluster center of mass, but rather in the outskirts. This is confirmed for four super spiral bright cluster galaxies (BCGs; OGC 0170, 0345, 1268, and 1304) recently observed with XMM-Newton by Bogdan et al. (2018), which turn out to be located at large distances $(150-1100 \mathrm{kpc})$ from the X-ray centroids of their host clusters. However, one super lenticular that they observed (OGC 0073) does turn out to be located at the center of a low-mass $\left(M_{500}=10^{14} M_{\odot}\right)$ galaxy cluster.

The presence of some super spirals in clusters is consistent with their high mass in stars and likely high halo mass. Super spirals may form preferentially in regions of the universe with relatively high overdensities, where a lot of gas is available to accrete onto their dark matter halos. On the other hand, a location at the periphery rather than the center of clusters is consistent with the morphology-density relation. We would not expect super spiral galaxies to survive as long as they have if they were at the centers of massive clusters, where they would be subject to frequent harassment by cluster galaxies.

\section{Galaxy Mergers}

Many super spirals and super lenticulars (41\%) appear to be involved in mergers (Table 9). There are 14 candidate interacting pairs or triples with projected separations of $28-131 \mathrm{kpc}$ and 27 advanced mergers with either double nuclei or overlapping disks with projected separations between 4 and $32 \mathrm{kpc}$. Only three of these pairs are spectroscopically confirmed (OGC 0044, 0299, and 0984). The remaining double nuclei, double disks, and interacting companions need to be verified spectroscopically to rule out chance alignments, but their appearance is quite suggestive of dynamical interactions. Many of them are highly distorted or have tidal tails or debris that confirm their merger status. We find seven candidate collisional ring galaxies (Table 9) and two galaxies with asymmetric, $\nu$-shaped arms (OGC 0290 and 1423). We find four additional galaxies with three or more arms that we do not classify as mergers (OGC 0256, 0926, 1046, and 1323), which may also have suffered a recent dynamical disturbance.

A high merger percentage is expected for galaxies as massive as super spirals and super lenticulars. Hopkins et al. (2010) estimate that galaxies with $M_{\text {stars }}=10^{12} M_{\odot}$ experienced on average 1.5 major mergers with mass ratio $\mu>0.3$ and 5.5 minor mergers with $0.1<\mu<0.3$ since $z=2$, based on halo occupation statistics. Most mergers experienced at late times by such massive galaxies are minor mergers because equally massive companions are quite rare. The fraction of massive galaxies with currently visible ongoing mergers can be estimated from the merger rates predicted by their model, multiplied by the merger visibility timescale. They predict a $\mu>0.1$ merger rate of $0.2 \mathrm{Gyr}^{-1}$ at $M_{\text {stars }}=10^{11} M_{\odot}$, increasing to $2 \mathrm{Gyr}^{-1}$ for $10^{12} M_{\odot}$ galaxies at $z=0.3$. For a merger visibility timescale of $1.0 \mathrm{Gyr}$, the corresponding merger fraction ranges from 0.2 to 1.0. This is roughly consistent with the observed super spiral merger fraction of 0.41 and merger rate of $0.4 \mathrm{Gyr}^{-1}$, for a median galaxy mass of $10^{11.6} M_{\odot}$. If the $11 / 14$ star-forming super post-mergers in our sample are also the product of super spiral major mergers, this gives a destructive major-merger fraction of 0.12 and rate of $0.12 \mathrm{Gyr}^{-1}$, assuming a post-merger settling timescale of $1.0 \mathrm{Gyr}$ (Lotz et al. 2008). During this settling time, obvious tidal signatures of the merger will disappear, and the $u-r$ color of the 
post-merger $u-r$ color will redden by 0.35 mag to $u-r=1.75$ mag (Figure 5), at which point it will be difficult to distinguish from other quenched giant ellipticals in our sample. Including 38 ongoing super spiral mergers and 11 star-forming super postmergers, and excluding super lenticular mergers, yields an overall star-forming merger fraction of 0.52 and merger rate of $0.5 \mathrm{Gyr}^{-1}$.

The ability of super spirals to survive under such a high merger rate depends on whether the mergers are major or minor, and also on the gas fraction (Hopkins et al. 2009). We find eight super spirals in major pairs $(\Delta g<1.19$ mag) with separations $<150 \mathrm{kpc}$ (Table 9), that are likely to undergo transformative major mergers in the next Gyr. The remaining 35 mergers are minor pairs or double nuclei galaxies whose disks survived (including three S0/Sa galaxies). It is difficult to estimate the progenitor mass ratio for the double nuclei galaxies, but they must have either been minor mergers or unusually gas-rich mergers in order to retain or re-form their disks. If they are minor mergers, then the ratio of major to minor mergers is 0.18 , roughly consistent with the $0.35-0.1$ major/minor merger ratio predicted by Hopkins et al. (2010) for galaxies with $M_{\text {stars }}=10^{11-12} M_{\odot}$. It will be necessary to obtain $\mathrm{H} \mathrm{I}$ and $\mathrm{CO}$ emission line measurements, or alternatively sub-mm dust continuum measurements, in order to determine the gas fractions of super spirals and its impact on their merger survivability.

\section{Discussion: Super Spiral Growth and Destruction}

Armed with an estimate of the merger rate (Section 7) and SSFRs (Section 3), measured at a median redshift of $z=0.22$, we can predict the space densities and masses in stars of super spiral progenitors at intermediate redshift $(z=1.0)$. Over that redshift interval (a period of $5.2 \mathrm{Gyr}$ ), both rates should increase by roughly a factor of 2, according the model of Hopkins et al. (2010), yielding an average merger rate of $1.3 \mathrm{Gyr}^{-1}$ and $1 / \mathrm{SSFR}=20.6 \mathrm{Gyr}$ along the ridge line of the SFMS. At this merger rate, super spirals will on average undergo 5.5 minor mergers with $\mu \sim 0.1$ and 1.2 major mergers with $\mu>0.3$ between $z=1.0$ and $z=0.22$. Roughly $70 \%$ of super spirals will be transformed into giant ellipticals or super lenticulars by major mergers over this redshift interval, reducing the comoving space density of super spirals by a factor of 3.3 and increasing the comoving space density of giant ellipticals by a factor of 1.3 .

Typical super spiral progenitors will increase their disk mass in stars by $33 \%$ from $z=1.0$ to the present via steady, in situ star formation. Super spirals that do not experience a major merger in this time will increase their mass in stars by another $69 \%$ from direct acquisition of stars through minor mergers, most of which will be incorporated into their halos and thick disks. Gas-rich minor mergers will also add gas to super spiral disks, which may be transformed into additional mass in stars via merger-induced starbursts. Assuming a gas fraction of 0.5 for the secondary galaxy, and that half of this gas is converted into stars, super spiral mass in stars will grow by an additional $35 \%$ from merger-induced starbursts, with most of this mass incorporated into the disk. Together with steady in situ star formation, this yields a combined $68 \%$ gain in disk mass in stars.

From this model, we predict that $70 \%$ of super spirals were destroyed by major mergers from $z=1.0$ to $z=0.2$ and transformed into giant ellipticals or super lenticulars. The remaining $30 \%$ that survived through this time period increased their total mass in stars by a factor of 2.4 , with half going to the disk and half going to the halo, while maintaining a relatively low $B / T$. The answer to the question of how super spirals survive is twofold. First, those that did survive are a factor of two less massive than giant ellipticals and therefore reside in regions that are on average a factor of two less dense, resulting in a lower overall merger rate. Second, their large masses protect them from mergers, such that $82 \%$ of all super spiral mergers at $z=0.2$ are minor mergers that do not destroy their disks.

\section{Conclusions}

We present a catalog of 84 SDSS super spirals, 15 super lenticulars, 14 post-mergers, and 1400 giant ellipticals, selected for $r$-band luminosity $L_{r}>8 L^{*}$ and redshift $z<0.3$. These galaxies represent the most massive galaxies in their redshift range, with masses in stars of $10^{11.3}-10^{12.3} M_{\odot}$. Super spirals are characterized by very large, high-mass, high-surfacebrightness, actively star-forming disks that fall on or below the SFMS of galaxies. Super lenticulars have low SSFRs, particularly red optical colors, and no discernible spiral arms. Super post-mergers may be the product of super spiral major mergers, caught during the quenching phase, before they have completely ceased star formation.

The location of super spirals in WISE-SDSS color space shows that their star-forming disks contain a mix of young and old stellar populations. Their WISE [4.6]-[12] colors are relatively blue compared to less-massive spirals because they have accumulated large masses of old stars in their disks, resulting from early formation in some of the most-massive dark matter halos. Super spiral disks are red on the inside and blue on the outside, consistent with ongoing growth and insideout formation by accretion of cold gas and minor mergers. Super spirals must form stars at a high rate throughout their lifetimes in order to grow their massive, gigantic disks and maintain their blue integrated colors.

Super disk galaxies are primarily found in moderate density environments, with on average half as many companions within $150 \mathrm{kpc}$, compared to giant ellipticals. For the $28 \%$ found in galaxy clusters, they are located at the cluster outskirts, with high relative velocities $\left(\sim 2000 \mathrm{~km} \mathrm{~s}^{-1}\right)$, consistent with the morphology-density relation. Star formation quenching is not an inevitable conclusion for the most-massive spiral galaxies, provided that they do not live at the dense centers of the largest galaxy clusters. Super spirals that do suffer major mergers may be transformed into super lenticulars or giant elliptical galaxies, providing a possible pathway to generate isolated giant elliptical galaxies outside of galaxy clusters.

A large percentage (41\%) of super disk galaxies are involved in ongoing mergers or interactions with other galaxies. We suggest that some super spirals survive because most mergers are minor mergers for such massive galaxies. Super spirals have low bulge/total luminosity ratios, also consistent with disk building by cold gas accretion and bulge construction by minor mergers. While a large reservoir of high-angular-momentum gas could also aid in preserving the structure of super spirals, it will require sensitive radio and sub-mm observations to establish whether or not this is actually the case.

Super spirals and super lenticulars are disk galaxy counterparts to the most-massive, giant elliptical galaxies. Star formation remains unquenched in most super spirals, in spite of their very large masses of old stellar populations. In fact, they appear to have survived through the ages in moderately dense 
environs, by virtue of their large masses. Anticipating future studies, the extreme masses, luminosities, and sizes of super spirals will open new parameter space for testing galaxy scaling laws and theories of massive galaxy formation and evolution. The large ongoing merger fraction and variety of merger mass ratios and geometries present in super spiral systems also provide a unique opportunity to study the impact of mergers on massive spiral galaxy structure, star formation, and evolution.

We thank Katey Alatalo for insightful discussions regarding the properties of post-merger and post-starburst galaxies. This work was made possible by the NASA/IPAC Extragalactic Database and the NASA/IPAC Infrared Science Archive, which are both operated by the Jet Propulsion Laboratory, California Institute of Technology, under contract with the National Aeronautics and Space Administration. We thank Ben Chan, Marion Schmitz, and the rest of the NED team for useful discussions and their support of this work. This publication makes use of data from the Galaxy Evolution Explorer and the Hubble Space Telescope, retrieved from the Mikulski Archive for Space Telescopes (MAST). STScI is operated by the Association of Universities for Research in Astronomy, Inc., under NASA contract NAS5-26555. Support for MAST for non-HST data is provided by the NASA Office of Space Science via grant NNX09AF08G and by other grants and contracts. Funding for the Sloan Digital Sky Survey IV has been provided by the Alfred P. Sloan Foundation, the U.S. Department of Energy Office of Science, and the Participating Institutions. SDSS acknowledges support and resources from the Center for High-Performance Computing at the University of Utah. The SDSS website is www.sdss.org. SDSS is managed by the Astrophysical Research Consortium for the Participating Institutions of the SDSS Collaboration. This publication makes use of data products from the Two Micron All Sky Survey, which is a joint project of the University of Massachusetts and the Infrared Processing and Analysis Center/California Institute of Technology, funded by the National Aeronautics and Space Administration and the National Science Foundation. This publication makes use of data products from the Wide-field Infrared Survey Explorer, which is a joint project of the University of California, Los Angeles, and the Jet Propulsion Laboratory/California Institute of Technology, funded by the National Aeronautics and Space Administration.

\section{Appendix}

\section{A.1. Catalog of the Most Optically Luminous Galaxies}

The Ogle et al. Galaxy Catalog (OGC) is presented in this Appendix (Table 2), including catalog names, SDSS $r$-band magnitude, $K$-corrected $r$-band luminosity, redshift, morphology, and spectral type. Catalog sources that we reject based on photometric contamination, bad redshifts, and spatial overlap with foreground or background sources are presented in Tables 3-5. Derived physical properties are presented for super spirals, lenticulars, and post mergers in Table 6. We present images of super spirals, lenticulars, and post-mergers at a scale of $150 \mathrm{kpc}$ on a side in Figures 11-13 and images of their larger $(300 \mathrm{kpc} \times 300 \mathrm{kpc})$ environs in Figures 14 and 15. Non-spiral galaxies with SEDs dominated by AGNs or with significant contamination by stars are listed in Table 7. Super spiral and lenticular associations with galaxy clusters and groups are given in Table 8, including cluster names, redshifts, galaxy counts, and separation. Candidate super spiral and lenticular mergers and pair separations are found in Table 9.

\section{A.2. Non-spiral AGNs and Quasi-stellar Object Hosts}

There are 12 non-spiral galaxies with SEDs contaminated by bright type-1 AGNs or stellar objects that we excluded from our analysis (Table 7 and Figure 13). Among these are six spectroscpically verified quasi-stellar object (QSO) host galaxies (OGC 0239, 0302, 0377, 0889, 1239, and 1245) and three known BL Lac hosts (OGC 0615, 0962, and 1229), where the AGNs may contribute significantly to the $r$-band luminosity. There are another three galaxies accompanied by bright stellar objects (OGC 0307, 0469, and 0646) which may be either stars or QSOs, for which no SDSS spectra are available. We originally identified QSO host OGC 0302 as a super spiral (Ogle et al. 2016), but HST imaging shows it to be a disturbed, possibly post-merger galaxy rather than a spiral galaxy (Figure 16).

\section{A.3. Gravitational Lenses}

Galaxy-scale gravitational lenses are potential contaminants to our sample that may artificially boost the $r$-band flux or present arc-like features that can be mistaken for spiral arms (Figure 16). For example, the giant elliptical galaxy OGC 0203 (2MASX J11125450 +1326093 in A1201) is a known gravitational lens, with a lens arc projected 2."0 from its center (Edge et al. 2003). We initially identified the previously unknown gravitational lens OGC 0200 (2MASX J08355126 +3926220 ) as a super spiral, but an existing HST image shows multiple lens arcs that masquerade as faint spiral arms in the lower-resolution SDSS images. Finally we identify the brightest cluster galaxy OGC 1565 (2MASX J21531028+1154551, $z=0.289$ ) as a gravitational lens candidate and possible Einstein ring by the unusual red ring that encircles it (Figure 16). 
Table 2

The Ogle et al. Galaxy Catalog (OGC)

\begin{tabular}{|c|c|c|c|c|c|}
\hline OGC & NED Name & $L_{r}^{\mathrm{a}}$ & $r(\mathrm{mag})^{\mathrm{b}}$ & $z(\mathrm{NED})^{\mathrm{c}}$ & Morph. $^{\mathrm{d}}$ \\
\hline 0021 & 2MASX J12220526+4518109 & 19.8 & 16.26 & 0.264357 & $\mathrm{E}$ \\
\hline 0022 & B3 $1715+425$ & 19.5 & 15.34 & 0.182900 & $\mathrm{E}$ \\
\hline 0023 & SDSS J215541.97+123128.5 & 19.1 & 15.68 & 0.193000 & $\mathrm{E}$ \\
\hline \multirow{2}{*}{$\begin{array}{l}\cdots \\
0078\end{array}$} & & & & & \\
\hline & 2MASX J02295551+0104361 & 13.3 & 16.53 & 0.245472 & E \\
\hline$\cdots$ & & & & & \\
\hline 1606 & SDSS J121644.34+122450.5 & 8.0 & 17.22 & 0.257144 & SS \\
\hline 1607 & SDSS J130454.84+100011.6 & 8.0 & 17.19 & 0.255875 & $\mathrm{E}$ \\
\hline 1608 & SDSS J040422.91-054134.9 & 8.0 & 17.27 & 0.250635 & SS \\
\hline 1609 & 2MASX J12071504+1713488 & 8.0 & 16.87 & 0.221788 & $\mathrm{E}$ \\
\hline 1610 & 2MASX J08302311+4744471 & 8.0 & 17.10 & 0.243735 & $\mathrm{E}$ \\
\hline 1611 & 2MASX J00380781-0109365 & 8.0 & 16.65 & 0.208695 & So \\
\hline $1662^{\mathrm{e}}$ & SDSS J085123.17-002148.7 & 7.9 & 17.59 & 0.295481 & Epec \\
\hline
\end{tabular}

Notes.

${ }^{a}$ SDSS $r$-band luminosity, $K$-corrected, and divided by $L^{*}=5.41 \times 10^{43} \mathrm{erg} \mathrm{s}^{-1}$.

${ }^{\mathrm{b}}$ SDSS $r$-band CModel magnitude.

${ }^{c}$ NED preferred redshift, primarily from SDSS DR6.

${ }^{\mathrm{d}}$ Galaxy morphology: $\mathrm{G}=$ unclassified galaxy, $\mathrm{E}=$ elliptical, Epec = peculiar elliptical, $\mathrm{S} 0=$ super lenticular, $\mathrm{S}=$ spiral, $\mathrm{SS}=$ super spiral.

e The starburst galaxy OGC 1662 falls below the $8.0 L^{*}$ cutoff of the sample (horizontal line), but we include it in this table, Table 6, and Figure 13 to point out its unusual properties.

(This table is available in its entirety in machine-readable form.)

Table 3

Luminous Galaxy Rejects: Inaccurate Photometry

\begin{tabular}{lll}
\hline \hline OGC & \multicolumn{1}{c}{ NED Name } & Notes \\
\hline 0001 & SDSS J101736.97+305101.6 & glare \\
0003 & SDSS J091428.98+184250.9 & glare \\
0004 & SDSS J102825.27+154757.2 & glare \\
$\ldots$ & & \\
1418 & 2MASX J14352497-0105084 & bright star \\
1425 & 2MASX J10240215+2534587 & glare \\
1585 & 2MASX J09095768+5731219 & bright star \\
\hline
\end{tabular}

(This table is available in its entirety in machine-readable form.)

Table 4

Luminous Galaxy Rejects: Incorrect Redshifts

\begin{tabular}{|c|c|c|c|c|c|}
\hline OGC & NED Name & $z$ (NED) & References (1) & Adopted $z$ & References (2) \\
\hline 0005 & 2MASX J14342221+0706510 & 0.254000 & RK12 & $\cdots$ & $\cdots$ \\
\hline 0006 & CGCG 045-047 & 0.192300 & HT10 & $\cdots$ & $\ldots$ \\
\hline 0008 & 2MASX J13041968+4214150 & 0.201700 & HT10 & 0.03611 & DR12 \\
\hline 0011 & UGC 08569 & 0.140657 & FK99 & 0.02303 & DR12 \\
\hline 0012 & 2MASX J16521103+2344396 & 0.178900 & HT10 & 0.03471 & DR12 \\
\hline 0013 & 2MASX J11064145+3435219 & 0.158800 & HT10 & $\ldots$ & $\ldots$ \\
\hline 0014 & LCSB S2225P & 0.222100 & RR05 & $\cdots$ & $\cdots$ \\
\hline 0015 & MCG +08-24-006 & 0.149600 & HT10 & $\cdots$ & $\cdots$ \\
\hline 0019 & SBS $0957+569$ & 0.147600 & HT10 & 0.01368 & DR12 \\
\hline 0020 & SDSS J082143.27+011423.2 & 0.295711 & GS09 & $\ldots$ & $\ldots$ \\
\hline 0024 & CGCG 208-020 & 0.132400 & HT10 & 0.02457 & DR12 \\
\hline 0026 & 2MASX J15032011+0856496 & 0.168000 & LS10 & $\ldots$ & $\cdots$ \\
\hline 0030 & 2MASX J01132663+1520072 & 0.203174 & CB09 & 0.04232 & DR12 \\
\hline 0036 & 2MASX J01122497+1538371 & 0.217106 & CB09 & 0.04423 & DR12 \\
\hline 0038 & 2MASX J14153929+2313477 & 0.252000 & BG06 & 0.06369 & DR12 \\
\hline 0045 & 2MASX J16064936+3202464 & 0.135400 & HT10 & 0.11550 & DR12 \\
\hline 0052 & VCC 0838 & 0.295417 & CG01 & $\ldots$ & $\ldots$ \\
\hline 0056 & KUG $1320+255$ & 0.143843 & RG01 & 0.03322 & DR12 \\
\hline 0069 & 2MASX J09075107+4401533 & 0.114500 & HT10 & 0.04878 & DR12 \\
\hline
\end{tabular}


Table 4

(Continued)

\begin{tabular}{lllcc}
\hline \hline OGC & NED Name & $z$ (NED) & References (1) & Adopted $z$ \\
\hline 0102 & SDSS J135128.05+091559.7 & 0.294330 & DR6 & 0.06062 \\
0172 & 2MASX J21472854-0738031 & 0.148299 & $6 \mathrm{dF}$ & 0.05971 \\
1035 & SDSS J141655.53+234018.2 & 0.281682 & DR13 & 0.11548 \\
1274 & UGC 10782 & 0.086000 & VC04 & 0.036 \\
1343 & 2MASX J12110100+3048346 & 0.192703 & DR6 & 0.12861 \\
\hline
\end{tabular}

References. DR6, 12, 13 = SDSS Data Release 6, 12, 13, 6dF = 6dF Galaxy Survey, BG06 = Barkhouse et al. (2006), CB09= Cava et al. (2009), CG01 = Conselice et al. (2001), FK99 = Falco et al. (1999), GS09 = Guzzo et al. (2009), HT10 = Hernandez-Toledo et al. (2010), LS10 = Lin et al. (2010), RG01 = Rines et al. (2001), RK12 = Rykoff et al. (2012), RR05 = Rowan-Robinson et al. (2005), VC04 = Veron-Cetty et al. (2004).

Table 5

Luminous Galaxy Rejects: Overlapping Objects

\begin{tabular}{lllll}
\hline \hline OGC & Object 1 (NED) & Type 1 & Object2 & Type 2 \\
\hline 0002 & SDSS J104819.41+123745.8 & compact & NGC 3384 & E \\
0010 & SDSS J104248.72+132710.7 & compact & UGC 05832 & Irr \\
0025 & SDSS J141309.29+083707.2 & compact & VV 299a & Irr \\
0031 & 2MASX J12494045+2546186 & compact & KUG 1247+260 & dE \\
0048 & 2MASX J07543650+3905307 & dE? & no name & compact \\
0059 & 2MASX J08540011+5751327 & E & UGC 04974 & S, edge-on \\
0146 & SDSS J092209.32+335057.4 & compact & SDSS J110717.65+080435.3 \\
0191 & VIII Zw 125 NOTES02 & QSO & SDSS J091031.83+330327.9 \\
0379 & KUG 0907+332 & E & SDSS J142418.71+293232.9 \\
0706 & SDSS J142418.44+293238.4 & E & no name & E \\
1176 & SDSS J144700.16+011504.9 & F9 star (cz $=9$ km s $\left.{ }^{-1}\right)$ & UGC 01225 NED02 & Irr \\
1322 & SDSS J014503.26-001800.0 & S & SDSS J115237.63+152759.1 \\
1360 & SDSS J123433.28+181154.8 & E & GALEXASC J020707.37-082723.5 \\
1406 & 2MASX J11523751+1527539 & E & no name & G \\
1497 & SDSS J020707.48-082726.0 & compact & SLr \\
1565 & 2MASX J21531028+1154551 & GLens candidate & Irr \\
\hline
\end{tabular}

Note. Galaxy morphology: compact $=$ star or compact galaxy, $\mathrm{G}=$ unclassified galaxy, $\mathrm{S}=$ spiral, $\mathrm{S} 0=$ lenticular, $\mathrm{E}=\mathrm{elliptical}$, $\mathrm{dE}=\mathrm{dwarf}$ elliptical, Irr = irregular galaxy, QSO = quasi-stellar object, GLens = gravitational lens.

Table 6

OGC Super Spirals, Lenticulars, and Post-mergers

\begin{tabular}{|c|c|c|c|c|c|c|c|c|}
\hline OGC & NED Name & $z(\operatorname{SDSS})^{\mathrm{a}}$ & $L_{r}^{\mathrm{b}}$ & $D^{\mathrm{c}}$ & $\log M_{\text {stars }}{ }^{\mathrm{d}}$ & $\log \mathrm{SFR}^{\mathrm{e}}$ & Morph. ${ }^{\mathrm{f}}$ & Spect. $^{\mathrm{g}}$ \\
\hline 0065 & 2MASX J10301576-0106068 & 0.28228 & 13.9 & 81 & 11.65 & 1.21 & bar & \\
\hline 0139 & 2MASX J16394598+4609058 & 0.24713 & 12.0 & 134 & 11.71 & 1.31 & $\cdots$ & $\mathrm{H} \alpha$ \\
\hline 0170 & 2MASX J10100707+3253295 & 0.28990 & 11.6 & 87 & 11.65 & 1.08 & bar & $\mathrm{H} \alpha$ \\
\hline 0179 & SDSS J213701.13-064447.0 & 0.29065 & 11.5 & 76 & 11.88 & 0.34 & $\cdots$ & $\cdots$ \\
\hline 0217 & 2MASX J13275756+3345291 & 0.24892 & 11.2 & 69 & 11.52 & 1.50 & bar & $\mathrm{H} \alpha, \mathrm{sb}$ \\
\hline 0222 & 2MASX J12220815+4844557 & 0.29861 & 11.1 & 95 & 11.78 & 0.44 & $\cdots$ & $\cdots$ \\
\hline 0256 & 2MASX J11593546+1257080 & 0.26353 & 10.9 & 87 & 11.56 & 0.90 & $\cdots$ & $\mathrm{H} \alpha$ \\
\hline 0290 & 2MASX J12343099+5156295 & 0.29592 & 10.6 & 62 & 11.55 & 1.26 & $\cdots$ & Sey1 \\
\hline 0293 & 2MASX J13044128+6635345 & 0.28862 & 10.6 & 77 & 11.74 & 0.69 & $\cdots$ & $\mathrm{H} \alpha$ \\
\hline 0299 & 2MASX J09094480+2226078 & 0.28539 & 10.5 & 83 & 11.84 & 0.65 & $\cdots$ & $\cdots$ \\
\hline 0306 & SDSS J122100.48+482729.1 & 0.29966 & 10.5 & 75 & 11.72 & 0.79 & $\cdots$ & $\cdots$ \\
\hline 0345 & 2MASX J09260805+2405242 & 0.22239 & 10.3 & 81 & 11.72 & 1.17 & $\cdots$ & $\mathrm{H} \alpha$ \\
\hline 0388 & 2MASX J17340613+6029190 & 0.27596 & 10.1 & 64 & 11.57 & 0.84 & $\cdots$ & $\cdots$ \\
\hline 0441 & SDSS J095727.02+083501.7 & 0.25652 & 9.9 & 88 & $\ldots$ & 0.81 & $\cdots$ & $\ldots$ \\
\hline 0454 & 2MASXi J1003568+382901 & 0.25860 & 9.9 & 56 & 11.68 & 1.28 & $\cdots$ & $\mathrm{H} \alpha, \mathrm{sb}$ \\
\hline 0516 & 2MASX J14475296+1447030 & 0.22069 & 9.7 & 95 & 11.65 & 0.89 & $\cdots$ & LINER \\
\hline 0543 & 2MASX J09470010+2540462 & 0.10904 & 9.6 & 99 & 11.65 & 1.05 & bar & Sey1 \\
\hline 0574 & SDSS J121148.70+662514.4 & 0.23789 & 9.5 & 63 & 11.75 & 0.96 & $\cdots$ & $\mathrm{H} \alpha$ \\
\hline 0586 & 2MASX J11535621+4923562 & 0.16673 & 9.5 & 90 & 11.63 & 1.27 & $\cdots$ & Sey 2 \\
\hline 0595 & 2MASX J07550424+1353261 & 0.22264 & 9.5 & 77 & 11.53 & 0.96 & bar & $\cdots$ \\
\hline 0612 & SDSS J093540.34+565323.8 & 0.29636 & 9.4 & 92 & 11.98 & $\cdots$ & bar & \\
\hline 0623 & 2MASX J09011007+2454570 & 0.25232 & 9.4 & 74 & 11.55 & 1.03 & $\cdots$ & Sey 1 \\
\hline 0637 & 2MASX J15575566+4322473 & 0.20641 & 9.3 & 80 & 11.60 & 0.72 & $\cdots$ & $\mathrm{H} \alpha$ \\
\hline
\end{tabular}


Table 6

(Continued)

\begin{tabular}{|c|c|c|c|c|c|c|c|c|}
\hline OGC & NED Name & $z(\mathrm{SDSS})^{\mathrm{a}}$ & $L_{r}^{\mathrm{b}}$ & $D^{\mathrm{c}}$ & $\log M_{\text {stars }}{ }^{\mathrm{d}}$ & $\log \mathrm{SFR}^{\mathrm{e}}$ & Morph. ${ }^{f}$ & Spect. $^{\mathrm{g}}$ \\
\hline 0696 & SDSS J102154.85+072415.5 & 0.29061 & 9.2 & 70 & $\cdots$ & 0.90 & $\cdots$ & $\mathrm{H} \alpha$ \\
\hline 0704 & 2MASX J03460305+0100064 & 0.18605 & 9.2 & 83 & 11.46 & 0.91 & $\ldots$ & $\cdots$ \\
\hline 0713 & 2MASX J08265512+1811476 & 0.26545 & 9.2 & 82 & 11.64 & 0.93 & bar & $\cdots$ \\
\hline 0749 & 2MASX J15591044+3826290 & 0.29073 & 9.1 & 70 & 11.56 & 0.98 & bar & Sey 1 \\
\hline 0753 & 2MASX J08022926+2325161 & 0.27152 & 9.1 & 66 & 11.35 & 0.98 & $\cdots$ & $\mathrm{H} \alpha$ \\
\hline 0755 & SDSS J113800.88+521303.9 & 0.29593 & 9.1 & 64 & 11.59 & 0.79 & $\ldots$ & $\ldots$ \\
\hline 0789 & 2MASX J08542169+0449308 & 0.15679 & 9.0 & 86 & 11.58 & 1.13 & bar & $\mathrm{H} \alpha$ \\
\hline 0799 & 2MASX J10472505+2309174 & 0.18256 & 9.0 & 72 & 11.65 & 1.01 & bar & \\
\hline 0800 & 2MASX J11191739+1419465 & 0.14377 & 9.0 & 71 & 11.55 & 0.98 & $\cdots$ & $\cdots$ \\
\hline 0804 & SDSS J135546.07+025455.8 & 0.23884 & 9.0 & 84 & $\ldots$ & 0.77 & & \\
\hline 0830 & SDSS J141754.96+270434.4 & 0.15753 & 9.0 & 69 & 11.69 & 0.90 & $\cdots$ & $\cdots$ \\
\hline 0909 & 2MASX J14381016+5030122 & 0.24665 & 8.8 & 66 & 11.53 & 0.32 & $\cdots$ & $\cdots$ \\
\hline 0926 & 2MASX J10304263+0418219 & 0.16092 & 8.8 & 70 & 11.66 & 1.36 & $\ldots$ & $\mathrm{H} \alpha$ \\
\hline 0928 & 2MASX J12374668+4812273 & 0.27245 & 8.8 & 66 & 11.46 & 1.08 & $\ldots$ & $\mathrm{H} \alpha$ \\
\hline 0968 & 2MASX J09312816+4424163 & 0.21940 & 8.7 & 65 & 11.50 & 1.29 & bar & Sey2, not a starburst \\
\hline 0975 & 2MASX J11410001+3848078 & 0.26770 & 8.7 & 72 & 11.54 & 1.08 & $\cdots$ & $\ldots$ \\
\hline 0983 & SDSS J153618.97+452246.8 & 0.23618 & 8.7 & 80 & 11.43 & 0.71 & $\cdots$ & $\ldots$ \\
\hline 0984 & SDSS J133737.88+494015.6 & 0.27233 & 8.7 & 73 & 11.74 & 0.35 & $\cdots$ & $\mathrm{H} \alpha$ \\
\hline 0995 & 2MASX J14440406+2029072 & 0.24820 & 8.7 & 76 & 11.83 & 0.31 & $\ldots$ & $\mathrm{H} \alpha$ \\
\hline 1023 & 2MASX J09254889+0745051 & 0.17227 & 8.6 & 68 & 11.62 & 0.85 & $\cdots$ & $\mathrm{H} \alpha$ \\
\hline 1046 & 2MASX J09362208+3906291 & 0.28293 & 8.6 & 70 & 11.42 & 0.85 & $\ldots$ & $\ldots$ \\
\hline 1088 & SDSS J140138.37+263527.6 & 0.28396 & 8.5 & 78 & $\ldots$ & 0.90 & & $\mathrm{H} \alpha$ \\
\hline 1094 & SDSS J163357.99+172839.5 & 0.26691 & 8.5 & 77 & $\ldots$ & 1.08 & & $\mathrm{H} \alpha$ \\
\hline 1107 & 2MASX J12072497-0150416 & 0.20957 & 8.5 & 69 & 11.46 & 0.91 & bar & $\ldots$ \\
\hline 1166 & 2MASX J22295446-0921345 & 0.27954 & 8.4 & 57 & 11.49 & 0.46 & $\ldots$ & \\
\hline 1182 & 2MASX J00495939-0853413 & 0.12181 & 8.4 & 66 & 11.84 & 0.82 & $\ldots$ & $\mathrm{H} \alpha$ \\
\hline 1196 & SDSS J154950.91+234444.1 & 0.26208 & 8.4 & 69 & $\cdots$ & 0.99 & $\cdots$ & $\mathrm{H} \alpha$ \\
\hline 1250 & 2MASX J12321515+1021195 & 0.16588 & 8.3 & 71 & 11.42 & 0.88 & $\cdots$ & $\cdots$ \\
\hline 1268 & 2MASX J12005393+4800076 & 0.27841 & 8.3 & 63 & 11.46 & 1.04 & $\ldots$ & $\mathrm{H} \alpha$ \\
\hline 1273 & 2MASX J07380615+2823592 & 0.23091 & 8.3 & 77 & 11.46 & 0.97 & bar & $\ldots$ \\
\hline 1287 & 2MASX J07404205+4332412 & 0.17828 & 8.3 & 69 & 11.62 & 0.90 & $\ldots$ & $\mathrm{H} \alpha$ \\
\hline 1304 & 2MASX J16014061+2718161 & 0.16440 & 8.3 & 82 & 11.80 & 1.33 & $\cdots$ & $\mathrm{H} \alpha$ \\
\hline 1312 & SDSS J143447.86+020228.6 & 0.27991 & 8.2 & 75 & 11.68 & 1.26 & $\cdots$ & $\mathrm{H} \alpha$, not a starburst \\
\hline 1323 & SDSS J112928.74+025549.9 & 0.23960 & 8.2 & 70 & 11.70 & 0.94 & bar & $\ldots$ \\
\hline 1329 & 2MASX J16273931+3002239 & 0.25990 & 8.2 & 86 & 11.75 & 0.48 & $\ldots$ & $\mathrm{H} \alpha$ \\
\hline 1337 & SDSS J093921.25+260709.8 & 0.27487 & 8.2 & 56 & 11.70 & 0.68 & $\ldots$ & $\mathrm{H} \alpha$ \\
\hline 1352 & SDSS J101603.97+303747.9 & 0.25191 & 8.2 & 69 & 12.00 & $\ldots$ & & $\mathrm{H} \alpha$ \\
\hline 1375 & 2MASX J00155012-1002427 & 0.17601 & 8.2 & 68 & 11.47 & 0.75 & $\cdots$ & $\mathrm{H} \alpha$ \\
\hline 1379 & 2MASX J09373465+1036552 & 0.17946 & 8.2 & 90 & 11.58 & 1.04 & bar & $\mathrm{H} \alpha$ \\
\hline 1395 & 2MASX J13103930+2235023 & 0.23123 & 8.1 & 66 & 11.53 & 0.86 & $\cdots$ & $\cdots$ \\
\hline 1409 & SDSS J151721.02+603302.6 & 0.28232 & 8.1 & 70 & 11.62 & 0.79 & $\ldots$ & $\mathrm{H} \alpha$ \\
\hline 1420 & 2MASX J13475962+3227100 & 0.22306 & 8.1 & 88 & 11.50 & 0.93 & $\ldots$ & $\cdots$ \\
\hline 1423 & SDSS J215250.41+122159.2 & 0.27310 & 8.1 & 61 & $\ldots$ & 0.90 & & $\mathrm{H} \alpha$ \\
\hline 1428 & 2MASX J11162790+3813476 & 0.23350 & 8.1 & 77 & 11.34 & 0.89 & $\cdots$ & $\cdots$ \\
\hline 1450 & SDSS J132743.82-031323.1 & 0.29502 & 8.1 & 64 & $\ldots$ & 0.91 & & $\mathrm{H} \alpha$ \\
\hline 1457 & 2MASX J09381666+1044508 & 0.23897 & 8.1 & 72 & 11.37 & 1.26 & $\ldots$ & $\mathrm{H} \alpha, \mathrm{sb}$ \\
\hline 1464 & 2MASX J10041606+2958441 & 0.29844 & 8.1 & 57 & 11.47 & 1.39 & $\cdots$ & $\mathrm{H} \alpha, \mathrm{sb}$ \\
\hline 1500 & 2MASX J10095635+2611324 & 0.24089 & 8.1 & 64 & 11.44 & 0.96 & $\cdots$ & $\mathrm{H} \alpha$ \\
\hline 1501 & 2MASX J09334777+2114362 & 0.17219 & 8.1 & 64 & 11.62 & 1.43 & $\cdots$ & QSO, not a starburst \\
\hline 1512 & SDSS J122944.64+272306.3 & 0.27573 & 8.0 & 101 & 11.48 & 0.89 & $\ldots$ & $\mathrm{H} \alpha$, not a starburst \\
\hline 1514 & SDSS J080317.08+325932.6 & 0.24848 & 8.0 & 55 & 11.89 & 0.24 & $\ldots$ & $\ldots$ \\
\hline 1520 & 2MASX J12354859+3919078 & 0.23706 & 8.0 & 63 & 11.64 & 1.46 & $\ldots$ & $\mathrm{H} \alpha, \mathrm{sb}$ \\
\hline 1544 & 2MASX J14472834+5908314 & 0.24551 & 8.0 & 68 & 11.58 & 0.80 & bar & $\mathrm{H} \alpha$ \\
\hline 1546 & 2MASX J13435549+2440484 & 0.13725 & 8.0 & 66 & 11.59 & 0.66 & $\ldots$ & $\mathrm{H} \alpha$ \\
\hline 1549 & 2MASX J08464747+0446053 & 0.24145 & 8.0 & 76 & 12.01 & $\ldots$ & $\ldots$ & $\cdots$ \\
\hline 1554 & 2MASX J13422833+1157345 & 0.27873 & 8.0 & 57 & 11.53 & 0.94 & $\ldots$ & $\mathrm{H} \alpha$ \\
\hline 1559 & CGCG $122-067$ & 0.08902 & 8.0 & 81 & 11.71 & 0.93 & & $\mathrm{H} \alpha$ \\
\hline 1562 & SDSS J163202.04+464545.7 & 0.29491 & 8.0 & 67 & 11.64 & 0.61 & $\ldots$ & $\mathrm{H} \alpha$ \\
\hline 1600 & SDSS J115155.92+104634.7 & 0.28305 & 8.0 & 67 & 11.63 & 0.22 & $\ldots$ & $\mathrm{H} \alpha$ \\
\hline 1606 & SDSS J121644.34+122450.5 & 0.25694 & 8.0 & 78 & $\ldots$ & 0.95 & bar & Sey1 \\
\hline 1608 & SDSS J040422.91-054134.9 & 0.25055 & 8.0 & 80 & 11.51 & 0.78 & $\cdots$ & $\mathrm{H} \alpha$ \\
\hline 0044 & 2MASX J14072225+1352512 & 0.29372 & 15.1 & 85 & 11.92 & 0.47 & $\mathrm{~S} 0 / \mathrm{Sa}$ & $\ldots$ \\
\hline 0073 & 2MASX J10405643-0103584 & 0.25024 & 13.4 & 82 & 11.81 & 0.49 & $\mathrm{~S} 0 / \mathrm{Sa}$ & $\ldots$ \\
\hline
\end{tabular}


Table 6

(Continued)

\begin{tabular}{|c|c|c|c|c|c|c|c|c|}
\hline OGC & NED Name & $z(\mathrm{SDSS})^{\mathrm{a}}$ & $L_{r}^{\mathrm{b}}$ & $D^{\mathrm{c}}$ & $\log M_{\text {stars }}{ }^{\mathrm{d}}$ & $\log \mathrm{SFR}^{\mathrm{e}}$ & Morph. ${ }^{f}$ & Spect. $^{\mathrm{g}}$ \\
\hline 0265 & SDSS J115052.98+460448.1 & 0.28946 & 10.8 & 88 & 11.80 & 0.10 & S0/Sa & $\ldots$ \\
\hline 0280 & 2MASX J09572689+4918571 & 0.24144 & 10.7 & 106 & 11.81 & 0.60 & S0/Sa & $\cdots$ \\
\hline 0425 & 2MASX J21160443-0702228 & 0.19082 & 10.0 & 77 & 11.85 & 0.60 & So/Sa & $\mathrm{H} \alpha$ \\
\hline 0581 & 2MASX J13423113+0021440 & 0.24342 & 9.5 & 142 & 11.80 & 0.64 & S0/Sa & $\cdots$ \\
\hline 1002 & 2MASX J10535662+5909155 & 0.19896 & 8.7 & 84 & 11.69 & 0.76 & S0/Sa & $\mathrm{H} \alpha$ \\
\hline 1220 & 2MASX J08164326+4702216 & 0.29529 & 8.3 & 62 & 11.55 & 0.63 & $\mathrm{~S} 0 / \mathrm{Sa}$ & $\mathrm{H} \alpha$ \\
\hline 1270 & SDSS J125157.99+305422.3 & 0.23065 & 8.3 & 78 & 11.77 & 0.48 & $\mathrm{~S} 0 / \mathrm{Sa}$ & $\mathrm{H} \alpha$ \\
\hline 1291 & SDSS J090317.22-000758.9 & 0.29726 & 8.3 & 72 & 11.77 & 0.33 & So/Sa & $\mathrm{H} \alpha$ \\
\hline 1381 & 2MASX J08093749+2316385 & 0.27291 & 8.2 & 81 & 11.65 & 0.33 & S0/Sa & $\cdots$ \\
\hline 1386 & 2MASX J13382172+0929423 & 0.24302 & 8.2 & 92 & 11.59 & 0.68 & S0/Sa & $\cdots$ \\
\hline 1526 & 2MASX J11414166+0223211 & 0.23354 & 8.0 & 89 & 11.58 & 0.39 & $\mathrm{~S} 0 / \mathrm{Sa}$ & $\mathrm{H} \alpha$ \\
\hline 1535 & 2MASX J11160517+3303477 & 0.20616 & 8.0 & 94 & 11.65 & 0.36 & So/Sa & $\cdots$ \\
\hline 1611 & 2MASX J00380781-0109365 & 0.20828 & 8.0 & 84 & 11.70 & 0.74 & $\mathrm{~S} 0 / \mathrm{Sa}$ & $\mathrm{H} \alpha$ \\
\hline 0247 & SDSS J081953.52+041409.2 & 0.296625 & 10.9 & 43 & 11.56 & 0.90 & Pec & $\mathrm{K}+\mathrm{A}+$ Sey 1 \\
\hline 0331 & SDSS J091318.25+492556.3 & 0.296414 & 10.4 & 43 & 11.52 & 0.80 & Pec & $\mathrm{K}+\mathrm{A}+$ Sey 1 \\
\hline 0624 & 2MASX J13245634+6219585 & 0.237397 & 9.4 & 69 & 11.64 & 1.06 & Pec & $\mathrm{K}+\mathrm{A} ?+$ Sey 1 \\
\hline 0707 & 2MASX J11304267+1538467 & 0.298136 & 9.2 & 61 & 11.50 & 0.60 & Pec & $\mathrm{K}+\mathrm{A}$ \\
\hline 0783 & SDSS J102629.10+094519.7 & 0.262280 & 9.0 & 72 & 11.69 & 0.82 & Pec & $\mathrm{K}+\mathrm{A}$ \\
\hline 0892 & SDSS J095543.25+111715.9 & 0.299029 & 8.8 & 66 & 11.70 & 0.18 & Pec & $\mathrm{H} \alpha$ \\
\hline 0902 & 2MASX J23591456+1351308 & 0.247131 & 8.8 & 55 & 11.56 & 1.34 & Pec & $\mathrm{H} \alpha, \mathrm{sb}$ \\
\hline 0973 & 2MASX J13412783+2851280 & 0.294948 & 8.7 & 47 & 11.59 & 1.03 & Pec & $\mathrm{K}+\mathrm{A}+\mathrm{H} \alpha$ \\
\hline 1056 & SDSS J120050.60-012755.6 & 0.267160 & 8.6 & 117 & $\ldots$ & 0.57 & Pec & \\
\hline 1058 & 2MASX J12383963+6413430 & 0.265032 & 8.6 & 82 & 11.57 & 1.21 & Pec & $\mathrm{H} \alpha, \mathrm{sb}$ \\
\hline 1174 & 2MASX J11310763+0224271 & 0.257493 & 8.4 & 65 & 11.50 & 0.88 & Pec & $\mathrm{H} \alpha$ \\
\hline 1377 & SDSS J134719.23+114915.1 & 0.279850 & 8.2 & 66 & 11.46 & 0.77 & Pec & $\mathrm{K}+\mathrm{A}+$ Sey 2 \\
\hline 1413 & MCG $+09-25-047$ & 0.244470 & 8.1 & 67 & 11.85 & $<1.90$ & Pec & $\mathrm{K}+\mathrm{A}+$ Sey 2 \\
\hline 1490 & 2MASX J08164043+3340182 & 0.238297 & 8.1 & 58 & 11.34 & 0.88 & Pec & $\mathrm{K}+\mathrm{A}+[\mathrm{N} \mathrm{II}]$ \\
\hline 1662 & SDSS J085123.17-002148.7 & 0.295481 & 7.9 & 72 & $\ldots$ & 1.19 & Pec & $\mathrm{H} \alpha, \mathrm{sb}$ \\
\hline
\end{tabular}

Notes.

${ }^{\text {a }}$ SDSS DR9 redshift.

${ }^{\mathrm{b}} L / L^{*}$ (Sloan $r$ band).

${ }^{\mathrm{c}}$ Isophotal diameter (kpc) at $r=25.0 \mathrm{mag} \operatorname{arcsec}^{-2}$.

d $\log$ of mass in stars $\left(M_{\odot}\right)$.

${ }^{\mathrm{e}} \log$ of star formation rate $\left(M_{\odot} \mathrm{yr}^{-1}\right)$.

${ }^{\mathrm{f}}$ Morphology. Lenticular galaxies are denoted S0/Sa. Galaxies with stellar bars are indicated as such. Horizontal lines in the table separate super spirals, super lenticulars, and super post-mergers.

${ }^{\mathrm{g}}$ Notes on SDSS spectroscopy. H $\alpha$ indicates detection of that line in the SDSS spectrum. AGNs are marked as Seyfert 1 (Sey1), Seyfert 2 (Sey2), LINER, or QSO. Galaxies with nuclear starbursts are marked $\mathrm{sb} . \mathrm{K}+\mathrm{A}$ indicates a post-starburst spectrum dominated by A stars. 


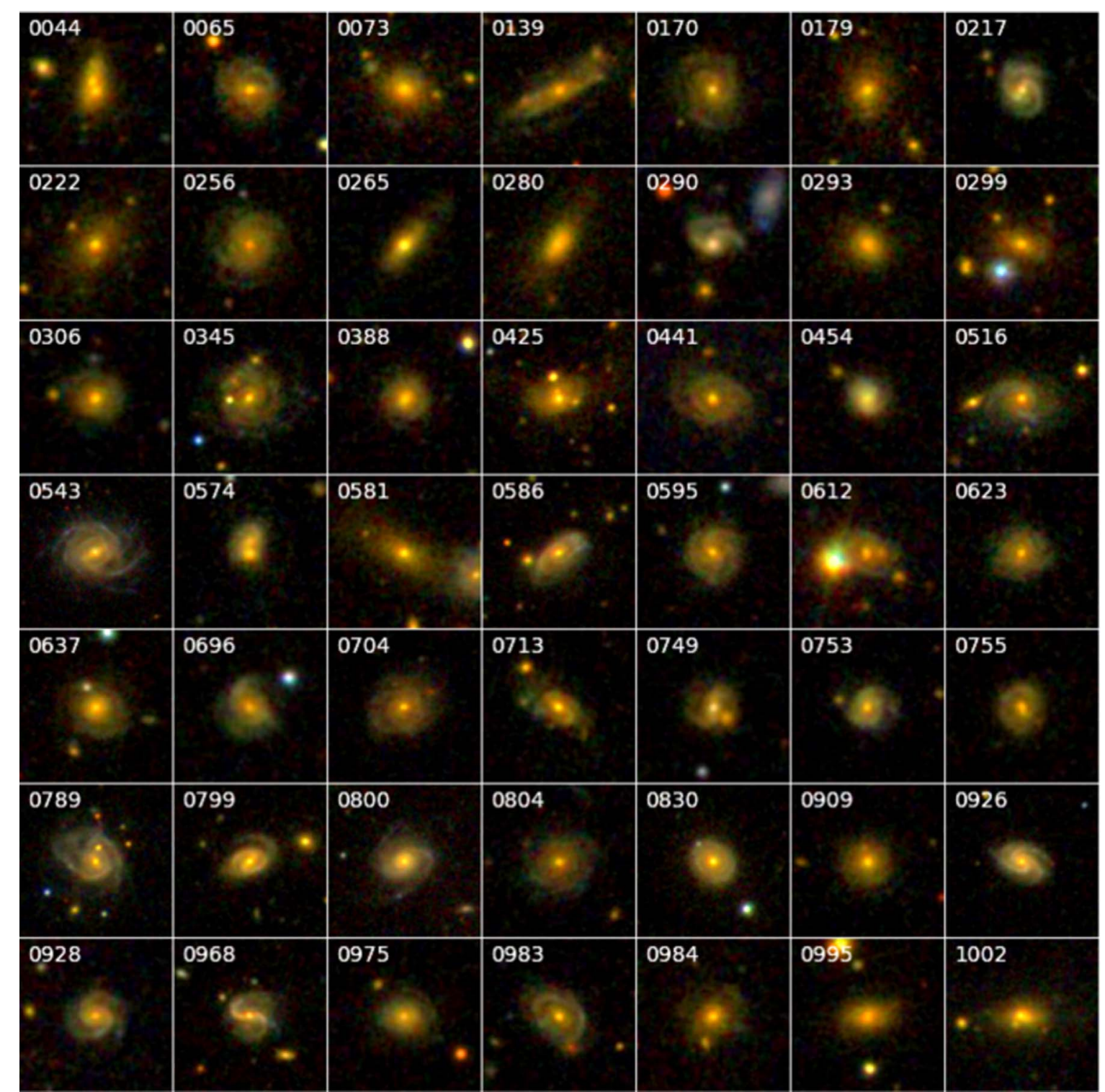

Figure 11. SDSS images of super spiral and super lenticular galaxies OGC 0044-1002, ordered by decreasing $r$-band luminosity. Each image is 150 kpc on a side. 


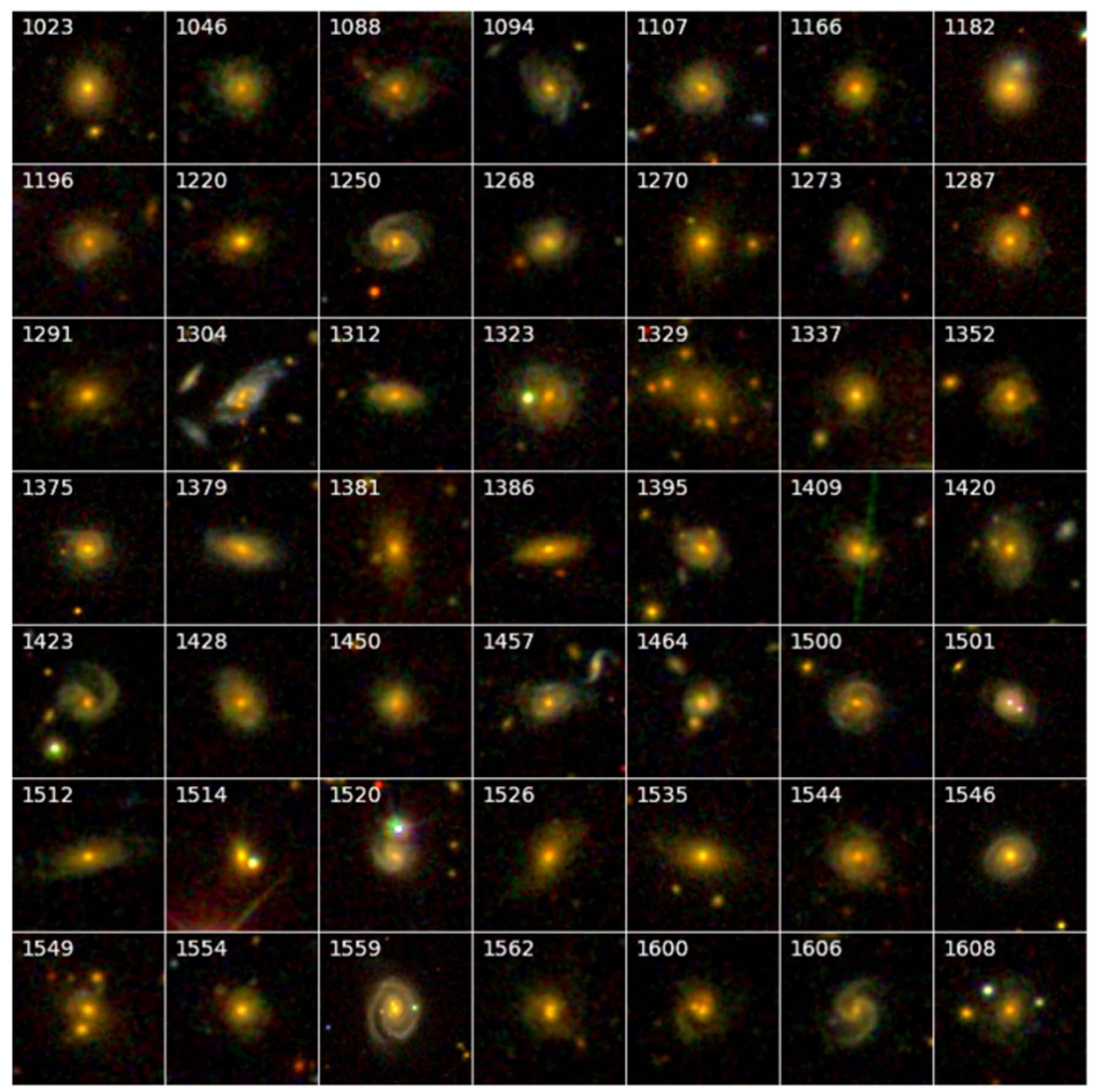

Figure 12. SDSS images of super spiral and super lenticular galaxies OGC 1023-1608, ordered by decreasing $r$-band luminosity. Each image is 150 kpc on a side. 


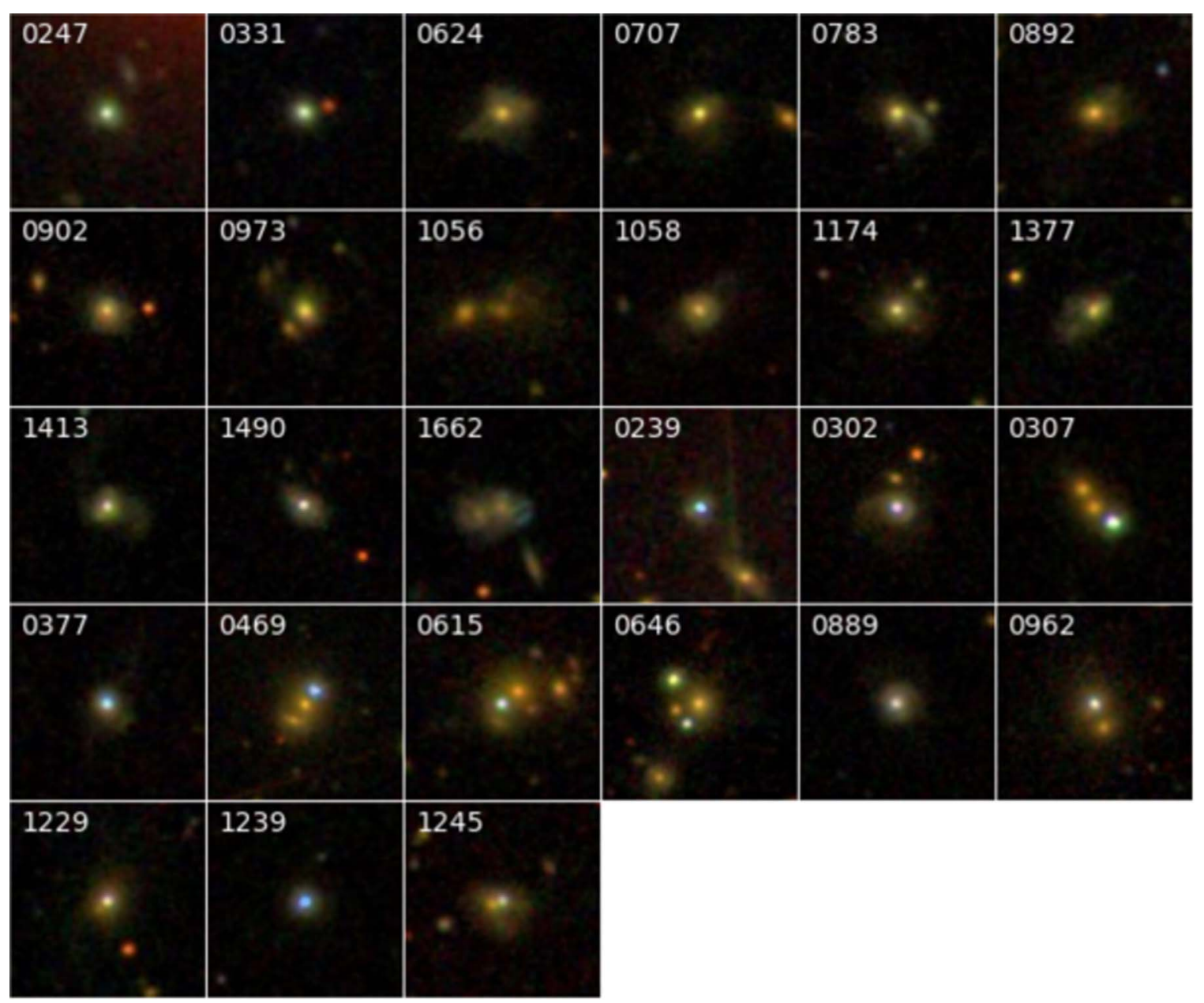

Figure 13. SDSS images (150 kpc on a side) of super post-mergers (OGC 0247-1662), followed by non-spiral quasi-stellar objects, or BL Lac hosts, or galaxies with stellar companions (OGC 0239-1245). 


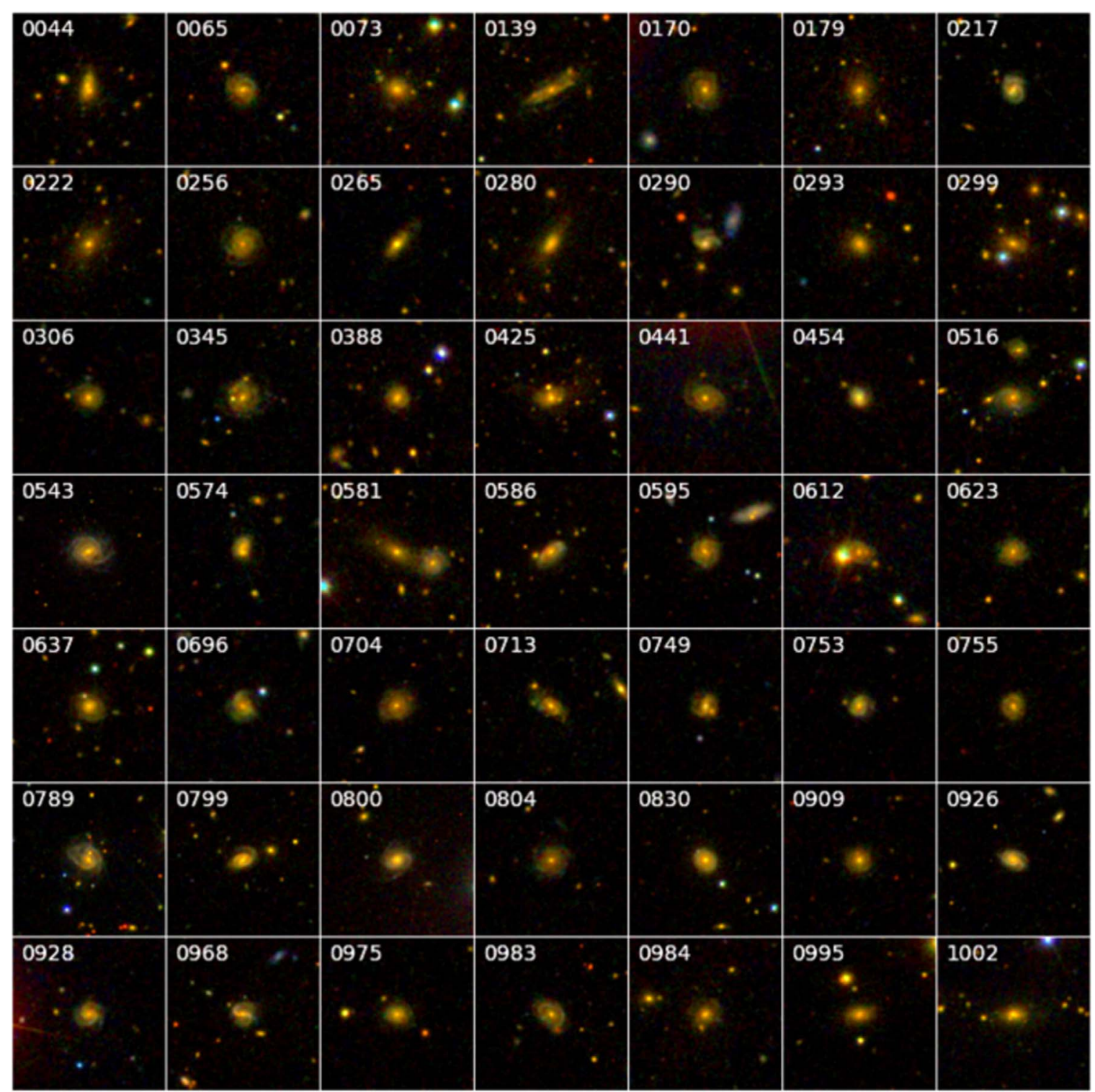

Figure 14. SDSS images of the environs of super spiral and super lenticular galaxies OGC 0044-1002, ordered by decreasing $r$-band luminosity. Each field of view is $300 \mathrm{kpc}$ on a side. 


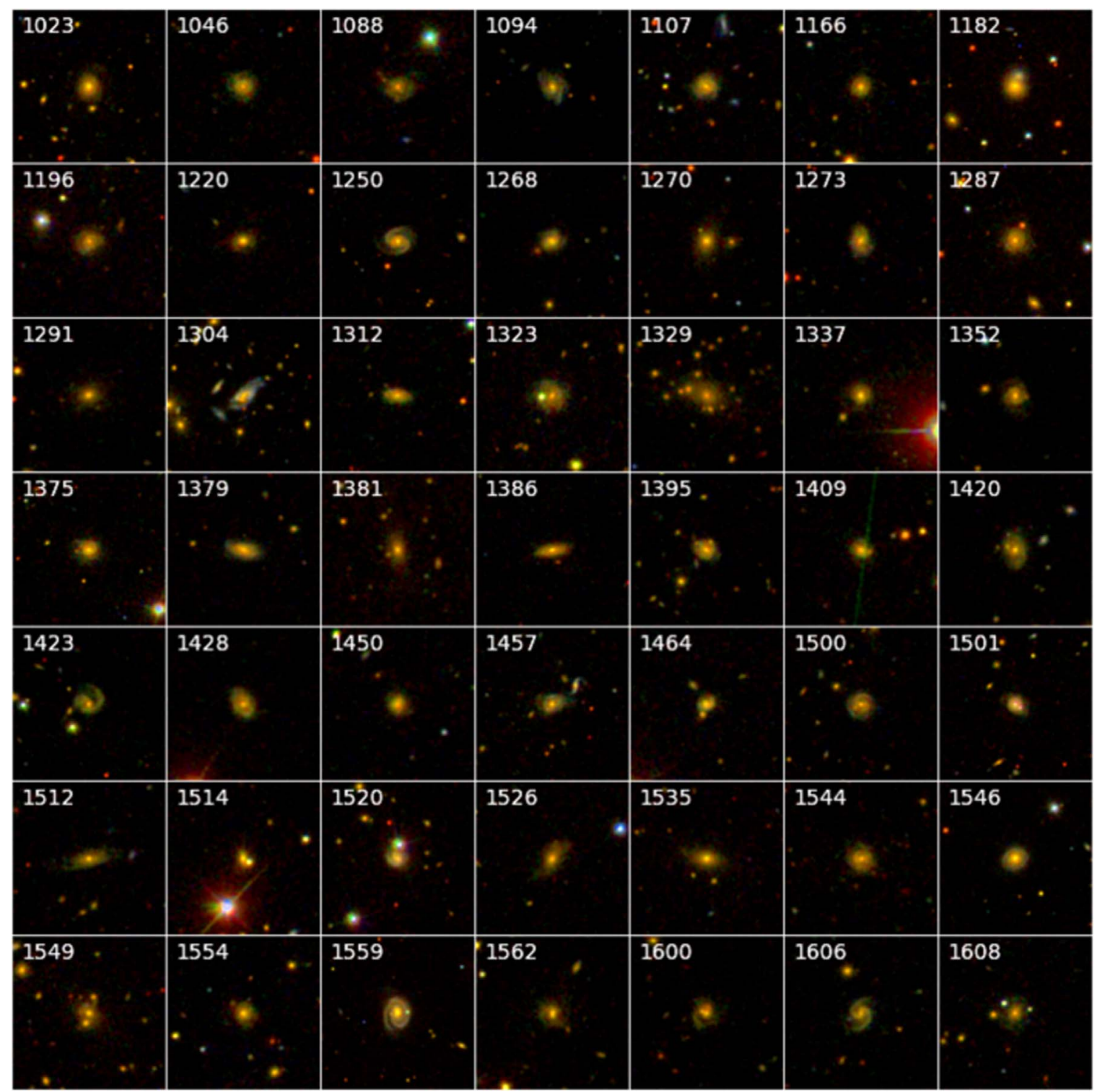

Figure 15. SDSS images of the environs of super spiral and super lenticular galaxies OGC 1023-1608, ordered by decreasing $r$-band luminosity. Each field of view is $300 \mathrm{kpc}$ on a side.

Table 7

OGC AGN-dominated

\begin{tabular}{lccc}
\hline \hline OGC & NED Name & Other Name & Image \\
\hline 0239 & 2MASX J11552373+1507564 & $\ldots$ & AGN host \\
0302 & 2MASX J15430777+1937522 & $\ldots$ & AGN host \\
0307 & SDSS J143335.34+242039.2 & $\ldots$ & $2 \mathrm{E}+$ star? \\
0377 & 2MASSi J2342593+134750 & $\ldots$ & AGN host \\
0469 & SDSS J150022.77+220027.3 & NVSS J150022+220027 & QSO \\
0615 & 2MASXi J0837247+145819 & ABELL 0689:[REE2012] BCG & $\ldots$ \\
0646 & 2MASX J21193928+1039326 & $\ldots$ & AGN host \\
0889 & 2MASX J08250928+2634381 & $\ldots$ & $\ldots$ \\
0962 & SDSS J141756.67+254326.2 & $\ldots$ & AGN \\
1229 & 2MASX J08574977+0135301 & $\ldots$ & AGN host \\
1239 & SDSS J145608.63+380038.5 & $\ldots$ & AGN host \\
1245 & 2MASX J02354663-0742506 & $\ldots$ & AGN host \\
\hline
\end{tabular}



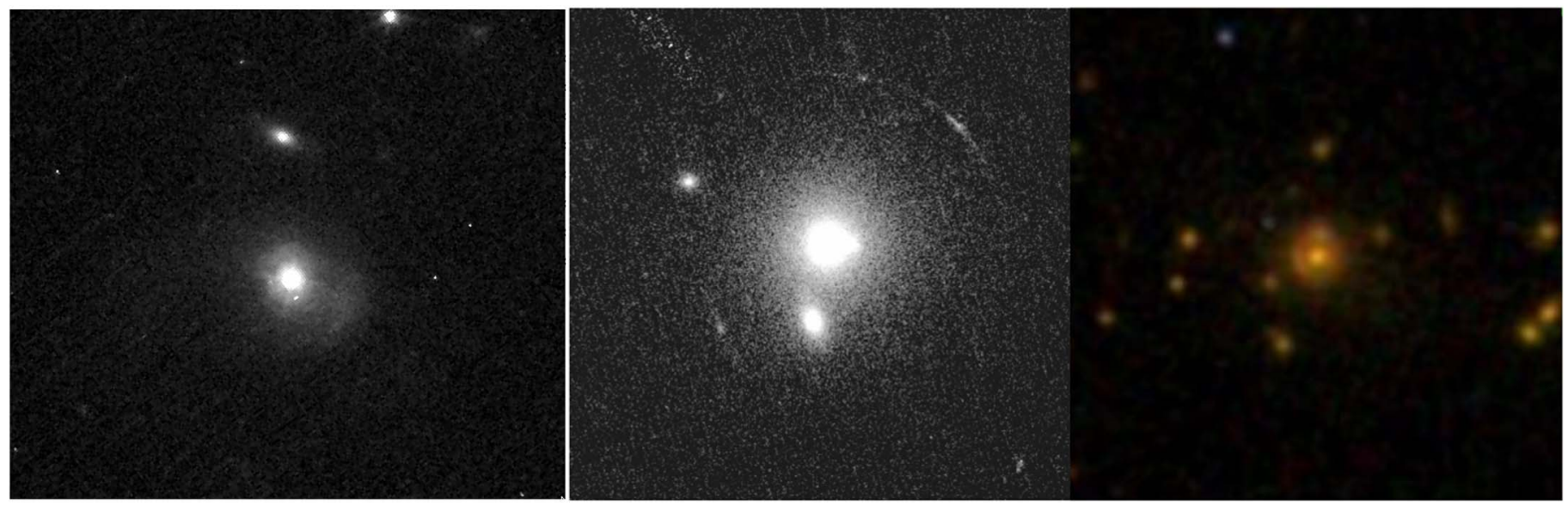

Figure 16. Left: HST WFPC2 F814W image of ex-super spiral OGC 0302, showing that it is a disturbed, non-spiral quasar host. The field of view is $24 \times 21$ arcsec $(88 \times 77 \mathrm{kpc})$. Center: elliptical galaxy and newly identified gravitational lens OGC 0200 (2MASX J08355126+3926220) imaged by HST ACS/HRC in the F775W filter (Proposal ID 10199). We initially classified this as a super spiral, mistaking the lens arcs for spiral arms. The field of view is $14 \times 14 \mathrm{arcsec}(56 \times 56 \mathrm{kpc})$. Right: newly identified gravitational lens candidate OGC 1565 (2MASX J21531028+1154551). The field of view is $50 \times 50 \operatorname{arcsec}(217 \times 217 \mathrm{kpc})$.

Table 8

Super Spiral and Lenticular Cluster and Group Associations

\begin{tabular}{|c|c|c|c|c|c|c|c|c|c|}
\hline OGC & NED Name & Redshift & $\mathrm{N} 1^{\mathrm{a}}$ & $\mathrm{N} 10^{\mathrm{b}}$ & Cluster Name & Type & Redshift & ztype $^{\mathrm{c}}$ & $\operatorname{Sep}()^{\prime}$ \\
\hline 0044 & 2MASX J14072225+1352512 & 0.293596 & 1 & 23 & GMBCG J211.84274+13.88070 & GClstr & 0.280850 & PHOT & 0.000 \\
\hline 0073 & 2MASX J10405643-0103584 & 0.250303 & 1 & 13 & SDSS CE J160.241898-01.069106 & GClstr & 0.254019 & EST & 0.013 \\
\hline 0170 & 2MASX J10100707+3253295 & 0.289913 & 2 & 38 & GMBCG J152.52936+32.89139 & GClstr & 0.319000 & PHOT & 0.001 \\
\hline 0179 & SDSS J213701.13-064447.0 & 0.290697 & 1 & 8 & SDSSCGB 18956 & GGroup & 0.291000 & SPEC & 0.054 \\
\hline 0280 & 2MASX J09572689+4918571 & 0.241492 & 5 & 26 & MaxBCG J149.36205+49.31591 & GClstr & 0.237650 & PHOT & 0.000 \\
\hline 0293 & 2MASX J13044128+6635345 & 0.288630 & 1 & 4 & MaxBCG J196.17181+66.59301 & GClstr & 0.226850 & PHOT & 0.000 \\
\hline 0299 & 2MASX J09094480+2226078 & 0.285386 & 2 & 17 & GMBCG J137.43670+22.43538 & GClstr & 0.303000 & PHOT & 0.000 \\
\hline 0345 & 2MASX J09260805+2405242 & 0.222451 & 1 & 36 & WHL J092608.1+240524 & GClstr & 0.178000 & PHOT & 0.000 \\
\hline 0388 & 2MASX J17340613+6029190 & 0.275807 & 1 & 2 & SDSSCGB 59704 & GGroup & 0.276000 & SPEC & 0.450 \\
\hline \multirow[t]{3}{*}{0516} & 2MASX J14475296+1447030 & 0.220592 & 1 & 33 & ABELL 1971 & GClstr & 0.208600 & SPEC & 1.298 \\
\hline & & & & & MaxBCG J221.98726+14.75906 & GClstr & 0.216050 & PHOT & 1.775 \\
\hline & & & & & WHL J144756.9+144532 & GClstr & 0.203600 & PHOT & 1.778 \\
\hline \multirow[t]{2}{*}{0581} & 2MASX J13423113+0021440 & 0.243520 & 1 & 47 & SDSSCG 110 & GGroup & 0.243400 & SPEC & 0.439 \\
\hline & & & & & SDSS CE J205.645691+00.368013 & GClstr & 0.231327 & EST & 1.031 \\
\hline 0586 & 2MASX J11535621+4923562 & 0.166892 & 3 & 70 & OGC 0586 CLUSTER & GClstr & 0.166187 & SPEC & 0.000 \\
\hline 0612 & SDSS J093540.34+565323.8 & 0.296393 & 1 & 21 & $\mathrm{ZwCl} 0932.1+5708$ & GClstr & $\ldots$ & $\ldots$ & 1.186 \\
\hline 0637 & 2MASX J15575566+4322473 & 0.206452 & 1 & 18 & MaxBCG J239.48210+43.37988 & GClstr & 0.202550 & PHOT & 0.000 \\
\hline 0704 & 2MASX J03460305+0100064 & 0.186147 & 1 & 167 & WHL J034603.0+010006 & GClstr & 0.181200 & PHOT & 0.008 \\
\hline 0755 & SDSS J113800.88+521303.9 & 0.296018 & 1 & 16 & SDSSCGB 65403 & GGroup & $\ldots$ & $\ldots$ & 1.104 \\
\hline 1002 & 2MASX J10535662+5909155 & 0.198533 & 1 & 26 & WHL J105356.6+590915 & GClstr & 0.210650 & PHOT & 0.001 \\
\hline \multirow[t]{3}{*}{1023} & 2MASX J09254889+0745051 & 0.172306 & 2 & 22 & GMBCG J141.45380+07.75151 & GClstr & 0.129650 & PHOT & 0.000 \\
\hline & & & & & MSPM 09586 & GClstr & 0.134320 & SPEC & 1.323 \\
\hline & & & & & SDSSCGA 00090 & GGroup & 0.134000 & SPEC & 1.872 \\
\hline 1088 & SDSS J140138.37+263527.6 & 0.284036 & 1 & 2 & $\mathrm{ZwCl} 1359.5+2650$ & GClstr & $\ldots$ & $\ldots$ & 1.902 \\
\hline 1166 & 2MASX J22295446-0921345 & 0.279639 & 1 & 2 & MaxBCG J337.47710-09.35962 & GClstr & 0.237650 & PHOT & 0.000 \\
\hline 1268 & 2MASX J12005393+4800076 & 0.278617 & 2 & 47 & GMBCG J180.22479+48.00211 & GClstr & 0.252000 & PHOT & 0.001 \\
\hline 1270 & SDSS J125157.99+305422.3 & 0.230703 & 3 & 14 & MaxBCG J192.99166+30.90620 & GClstr & 0.283550 & PHOT & 0.001 \\
\hline \multirow[t]{3}{*}{1304} & 2MASX J16014061+2718161 & 0.164554 & 3 & 164 & GMBCG J240.41924+27.30444 & GClstr & 0.193000 & PHOT & 0.000 \\
\hline & & & & & MaxBCG J240.43568+27.30263 & GClstr & 0.164750 & PHOT & 0.883 \\
\hline & & & & & WHL J160144.6+271809 & GClstr & 0.162200 & PHOT & 0.892 \\
\hline 1329 & 2MASX J16273931+3002239 & 0.259761 & 1 & 19 & GMBCG J246.91981+30.01418 & GClstr & 0.261000 & PHOT & 1.588 \\
\hline 1420 & 2MASX J13475962+3227100 & 0.223113 & 1 & 16 & SDSSCGB 16827 & GGroup & $\ldots$ & $\ldots$ & 0.748 \\
\hline 1520 & 2MASX J12354859+3919078 & 0.237013 & 1 & 13 & SDSSCGB 36014 & GGroup & $\ldots$ & $\ldots$ & 1.743 \\
\hline 1549 & 2MASX J08464747+0446053 & 0.241509 & 1 & 20 & WHL J084647.5+044605 & GClstr & 0.237650 & PHOT & 0.000 \\
\hline 1559 & CGCG $122-067$ & 0.089008 & 5 & 310 & MSPM 05544 & GClstr & 0.089190 & SPEC & 0.001 \\
\hline
\end{tabular}

Notes.

${ }^{\mathrm{a}}$ Number of galaxies within $1 \mathrm{Mpc}$ and $500 \mathrm{~km} \mathrm{~s}^{-1}$.

${ }^{\mathrm{b}}$ Number of galaxies within $10 \mathrm{Mpc}$ and $5000 \mathrm{~km} \mathrm{~s}^{-1}$.

${ }^{c}$ Redshift type, from NED or reference therein. EST—estimated, PHOT—photometric, and SPEC—spectroscopic.

${ }^{\mathrm{d}}$ Separation (in arcminutes) of the cluster or group catalog position in NED from the super spiral or super lenticular. In many cases the separation is zero because the brightest galaxy (i.e., the super spiral) position was apparently used to define the cluster position. 
Table 9

Super Spiral and Lenticular Mergers and Interacting Pairs

\begin{tabular}{|c|c|c|c|c|c|c|}
\hline OGC & Merger & Features & Companion & $z_{2}$ & Sep. $(")$ & $\overline{\text { Sep. }(\mathrm{kpc})^{\mathrm{a}}}$ \\
\hline 0044 & 2 nuclei & $\ldots$ & SDSS J140722.29+135250.3 & 0.28601 & 2.0 & 9.0 \\
\hline 0290 & major pair & $\nu$ shape & SDSS J123429.74+515639.8 & $\ldots$ & 16.0 & 72.0 \\
\hline \multirow[t]{2}{*}{0299} & major triple & $\cdots$ & SDSS J090944.05+222605.4 & $\ldots$ & 11.0 & 46.0 \\
\hline & $\ldots$ & $\ldots$ & SDSS J090944.08+222632.2 & 0.28380 & 27.0 & 115.0 \\
\hline 0425 & 2 nuclei & debris & $\ldots$ & $\ldots$ & 2.0 & 6.0 \\
\hline \multirow[t]{2}{*}{0516} & major triple & $\cdots$ & SDSS J144753.90+144701.2 & $\cdots$ & 14.0 & 48.0 \\
\hline & $\ldots$ & $\ldots$ & SDSS J144752.76+144728.7 & $\ldots$ & 27.0 & 95.0 \\
\hline 0574 & 2 nuclei & $\ldots$ & 2MASX J12114871+6625146 & $\ldots$ & 3.0 & 12.0 \\
\hline 0581 & major pair & $\ldots$ & SDSS J134229.89+002138.1 & $\ldots$ & 19.0 & 74.0 \\
\hline 0612 & 2 nuclei & $\ldots$ & SDSS J093540.00+565324.0 & $\ldots$ & 3.0 & 13.0 \\
\hline 0749 & 2 nuclei & $\cdots$ & SDSS J155910.19+382626.9 & $\cdots$ & 4.0 & 15.0 \\
\hline 0755 & 2 nuclei & ring & $\ldots$ & $\ldots$ & 1.0 & 4.0 \\
\hline 0789 & 2 nuclei & tail & $\ldots$ & $\ldots$ & 5.0 & 14.0 \\
\hline 0799 & major pair & ring & SDSS J104723.75+230923.4 & $\ldots$ & 19.0 & 58.0 \\
\hline 0909 & 2 nuclei & $\cdots$ & $\ldots$ & $\cdots$ & 4.0 & 15.0 \\
\hline 0968 & 2 nuclei & $\ldots$ & $\ldots$ & $\ldots$ & 3.0 & 11.0 \\
\hline 0983 & 2 nuclei & ring & SDSS J153618.68+452238.8 & $\ldots$ & 9.0 & 32.0 \\
\hline 0984 & major pair & $\ldots$ & SDSS J133740.61+494023.5 & 0.27221 & 28.0 & 115.0 \\
\hline 1002 & 2 nuclei & $\ldots$ & $\ldots$ & $\ldots$ & 3.0 & 9.0 \\
\hline 1088 & 2 nuclei & tail & SDSS J140138.83+263530.6 & $\cdots$ & 7.0 & 30.0 \\
\hline 1094 & 2 disks & $\ldots$ & SDSS J163357.74+172836.1 & $\ldots$ & 5.0 & 20.0 \\
\hline 1182 & 2 nuclei & $\ldots$ & SDSS J004959.22-085332.0 & $\ldots$ & 10.0 & 21.0 \\
\hline 1196 & minor pair & ring & SDSS J154949.79+234452.2 & $\ldots$ & 17.0 & 69.0 \\
\hline 1250 & minor pair & & SDSS J123212.17+102121.9 & $\ldots$ & 44.0 & 125.0 \\
\hline 1304 & 2 nuclei & $\ldots$ & $\ldots$ & $\ldots$ & 3.0 & 9.0 \\
\hline \multirow[t]{2}{*}{1329} & nest & $\ldots$ & SDSS J162739.16+300217.7 & $\ldots$ & 7.0 & 28.0 \\
\hline & $\ldots$ & $\ldots$ & SDSS J162739.98+300227.5 & $\ldots$ & 9.0 & 38.0 \\
\hline 1352 & minor pair & $\ldots$ & SDSS J101605.14+303751.3 & $\cdots$ & 16.0 & 61.0 \\
\hline 1375 & 2 nuclei & tail & SDSS J001550.69-100243.4 & $\ldots$ & 8.0 & 25.0 \\
\hline 1395 & 2 nuclei & tail & SDSS J131039.16+223506.9 & $\cdots$ & 5.0 & 18.0 \\
\hline 1409 & 2 nuclei & ring & SDSS J151720.48+603301.9 & $\ldots$ & 4.0 & 17.0 \\
\hline 1420 & 2 nuclei & $\ldots$ & $\ldots$ & $\ldots$ & 5.0 & 17.0 \\
\hline 1423 & minor pair & $\nu$ shape & SDSS J215251.02+122156.1 & $\ldots$ & 10.0 & 40.0 \\
\hline 1457 & major pair & tail & SDSS J093815.80+104500.9 & $\ldots$ & 16.0 & 60.0 \\
\hline 1464 & 2 nuclei & $\ldots$ & SDSS J100416.17+295839.9 & $\cdots$ & 5.0 & 21.0 \\
\hline 1500 & 2 nuclei & ring & $\ldots$ & $\ldots$ & 4.0 & 14.0 \\
\hline 1501 & 2 nuclei & debris & SDSS J093347.52+211434.0 & $\ldots$ & 4.0 & 12.0 \\
\hline 1514 & 2 nuclei & $\ldots$ & SDSS J080316.86+325930.9 & $\ldots$ & 3.0 & 13.0 \\
\hline 1549 & 2 nuclei & debris & SDSS J084647.56+044559.9 & $\cdots$ & 5.0 & 20.0 \\
\hline 1554 & major pair & ring & SDSS J134227.34+115707.0 & $\cdots$ & 31.0 & 131.0 \\
\hline 1559 & 2 nuclei & debris & $\ldots$ & $\ldots$ & 4.0 & 6.0 \\
\hline 1562 & 2 nuclei & tail & $\ldots$ & $\ldots$ & 1.0 & 4.0 \\
\hline 1600 & 2 nuclei & tail & $\ldots$ & $\ldots$ & 2.0 & 9.0 \\
\hline 1608 & minor pair & $\ldots$ & SDSS J040423.64-054135.7 & $\cdots$ & 11.0 & 43.0 \\
\hline
\end{tabular}

Note.

${ }^{\mathrm{a}}$ Projected separation.

\section{ORCID iDs}

Patrick M. Ogle (1) https://orcid.org/0000-0002-3471-981X Lauranne Lanz (i) https://orcid.org/0000-0002-3249-8224 Philip N. Appleton (1) https://orcid.org/0000-0002-7607-8766

\section{References}

Alatalo, K., Bitsakis, T., Lanz, L., et al. 2017, ApJ, 843, 9

Alatalo, K., Cales, S. L., Appleton, P. N., et al. 2014, ApJL, 794, L13 Bamford, S. P., Nichol, R. C., Baldry, I. K., et al. 2009, MNRAS, 393, 1324 Barkhouse, W. A., Green, P. J., Vikhlinin, A., et al. 2006, ApJ, 645, 955 Bell, E. F., \& de Jong, R. S. 2000, MNRAS, 312, 497

Bell, E. F., McIntosh, D. H., Katz, N., \& Weinberg, M. D. 2003, ApJS, 149,289

Blanton, M. R., Hogg, D. W., Bahcall, N. A., et al. 2003, ApJ, 592, 819

Bogdan, A., Lovisari, L., Kovacs, O. E., et al. 2018, ApJ, 869, 105
Bressan, A., Panuzzo, P., Buson, L., et al. 2006, ApJL, 639, L55 Brinchmann, J., Charlot, S., White, S. D. M., et al. 2004, MNRAS, 351, 1151 Bruzual, G., \& Charlot, S. 2003, MNRAS, 344, 1000

Burns, J. O., Hallman, E. J., Brennan, G., Motl, P. M., \& Norman, M. L. 2008, ApJ, 675, 1125

Cava, A., Bettoni, D., Poggianti, B. M., et al. 2009, A\&A, 495, 707 Chabrier, G. 2003, PASP, 115, 763

Chang, Y.-Y., van der Wel, A., da Cunha, E., \& Rix, H.-W. 2015, ApJS, 219, 8 Conselice, C. J., Gallagher, J. S., III, \& Wyse, R. F. G. 2001, ApJ, 559, 791 da Cunha, E., Charlot, S., \& Elbaz, D. 2008, MNRAS, 339, 1595

Davari, R. H., Ho, L. C., Mobasher, B., \& Canalizo, G. 2017, ApJ, 836, 75 de Jong, R. S. 1996, A\&A, 313, 377

Dekel, K., \& Birnboim, Y. 2006, MNRAS, 368, 2

Edge, A. C., Smith, G. P., Sand, D. J., et al. 2003, ApJL, 599, L69

Egami, E., Misselt, K. A., Rieke, G. H., et al. 2006, ApJ, 647, 922

Eisenstein, D. J., Annis, J., Gunn, J. E., et al. 2001, AJ, 122, 2267

Elbaz, D., Daddi, E., Le Borgne, D., et al. 2007, A\&A, 468, 33

Faisst, A. L., Carollo, C. M., Capak, P. L., et al. 2017, ApJ, 839, 71 
Falco, E. E., Kurtz, M. J., Geller, M. J., et al. 1999, PASP, 111, 438 Fraser-McKelvie, A., Brown, M. J. I., Pimbblet, K. A., et al. 2016, MNRAS, 4612, L11

Governato, F., Willman, B., Mayer, L., et al. 2007, MNRAS, 374, 1479

Guzzo, L., Schuecker, P., Bohringer, H., et al. 2009, A\&A, 499, 357

Hernandez-Toledo, H. M., Vazquez-Mata, J. A., Martinez-Vazquez, L., Choi, Y. Y., \& Park, C. 2010, AJ, 139, 2525

Hopkins, P. F., Bundy, K., Croton, D., et al. 2010, ApJ, 715, 202

Hopkins, P. F., Cox, T. J., Younger, J. D., \& Hernquist, L. 2009, ApJ, 691, 1168 Hopkins, P. F., Hernquist, L., Cox, T. J., et al. 2006, ApJS, 163, 1

Lin, Y.-T., Shen, Y., Strauss, M. A., Richards, G. T., \& Lunnan, R. 2010, ApJ, 723,1119

Lintott, C. J., Schawinski, K., Bamford, S., et al. 2011, MNRAS, 410, 166

Lintott, C. J., Schawinski, K., Slosar, A., et al. 2008, MNRAS, 389, 1179

Lotz, J. M., Jonsson, P., Cox, T. J., \& Primack, J. R. 2008, MNRAS, 391, 1137

Martig, M., Bournaud, F., Teyssier, R., \& Dekel, A. 2009, ApJ, 707, 250

Masters, K. L., Mosleh, M., Romer, A. K., et al. 2010, MNRAS, 405, 783

Melnick, J., \& De Propris, R. 2013, MNRAS, 431, 2034

Ogle, P. M., Lanz, L., Nader, C., \& Helou, G. 2016, ApJ, 817, 109
Posti, L., Fraternali, F., \& Marasco, A. 2019, A\&A, 626, 56

Rines, K., Geller, M. J., Kurtz, M. J., et al. 2001, ApJ, 561, 41

Rodriguez-Gomez, V., Pillepich, A., Sales, L. V., et al. 2016, MNRAS, 458, 2371

Rowan-Robinson, M., Babbedge, T., Surace, J., et al. 2005, AJ, 129, 1183

Rykoff, E. S., Koester, B. P., Rozo, E., et al. 2012, ApJ, 746, 178

Schawinski, K., Urry, C. M., Simmons, B. D., et al. 2014, MNRAS, 440, 889

Schlafly, E. F., \& Finkbeiner, D. P. 2011, ApJ, 737, 103

Sheth, K., Elmegreen, D. M., Elmegreen, B. G., et al. 2008, ApJ, 675, 1141

Simard, L., Mendel, J. T., Patton, D. R., Ellison, S. L., \& McConnachie, A. W. 2011, ApJS, 196, 11

Skrutskie, M. F., Cutri, R. M., Stiening, R., et al. 2006, AJ, 131, 1163

Springel, V., \& Hernquist, L. 2005, ApJL, 622, L9

Strauss, M. A., Weinberg, D. H., Lupton, R. H., et al. 2002, AJ, 124, 1810

Tremonti, C. A., Heckman, T. M., Kauffmann, G., et al. 2004, ApJ, 613, 898

Veron-Cetty, M.-P., Balayan, S. K., Mickaelian, A. M., et al. 2004, A\&A, 414, 487

Wright, E. L., Eisenhardt, P. R. M., Mainzer, A. K., et al. 2010, AJ, 140, 1868

York, D. G., Adelman, J., Anderson, J. E. Jr., et al. 2000, AJ, 120, 1579 